Check for updates

Cite this: J. Mater. Chem. A, 2020, 8 , 22840

Received 25th August 2020

Accepted 13th October 2020

DOI: $10.1039 / \mathrm{d} 0 \operatorname{ta} 08351 \mathrm{~h}$

rsc.li/materials-a

\section{Morphology of Cu clusters supported on reconstructed polar ZnO (0001) and (0001) surfaces $\uparrow$}

\author{
Michael D. Higham, (D)*ab David Mora-Fonz, (D) c Alexey A. Sokol, (D) d \\ Scott M. Woodley (iD ${ }^{d}$ and C. Richard A. Catlow (iD) abd
}

\begin{abstract}
Unbiased Monte Carlo procedures are applied to investigate the structure of Cu clusters of various sizes deposited over reconstructed polar ZnO surfaces. Four distinct reconstructed polar ZnO surfaces (two Zn terminated (0001) reconstructions and two O terminated (0001) reconstructions) were investigated, having previously been determined to be the most stable under typical conditions, as revealed by the grand canonical ensemble studies. Random sampling was performed considering $\sim 400000$ random initial structural configurations of $\mathrm{Cu}$ atoms over the $\mathrm{ZnO}$ surfaces, with each structure being optimised using interatomic potential techniques, and the most stable resultant structures being refined using a plane-wave DFT approach. The investigation reveals the key role of surface adatoms and vacancies arising from the reconstruction of the polar $\mathrm{ZnO}$ surface in determining the morphology of deposited $\mathrm{Cu}$ clusters. Strong $\mathrm{Cu}-\mathrm{Zn}$ interactions play an essential role in $\mathrm{Cu}$ cluster growth, with reconstructed polar $\mathrm{ZnO}$ surfaces featuring sites with undercoordinated $\mathrm{Zn}$ surface atoms promoting highly localised three dimensional $\mathrm{Cu}$ cluster morphologies, whist reconstructions featuring undercoordinated $\mathrm{O}$ atoms tend to result in more planar $\mathrm{Cu}$ clusters, in order to maximise the favourable $\mathrm{Cu}-\mathrm{Zn}$ interaction. This is the first study that evaluates the thermodynamically most stable $\mathrm{Cu} / \mathrm{ZnO}$ structures using realistic reconstructed $\mathrm{ZnO}$ polar surfaces, and thus provides valuable insights into the factors affecting $\mathrm{Cu}$ cluster growth over $\mathrm{ZnO}$ surfaces, as well as model catalyst surfaces that can be utilised in future computational studies to explore catalytic activity for key processes such as $\mathrm{CO}_{2}$ and $\mathrm{CO}$ hydrogenation to methanol.
\end{abstract}

\section{Introduction}

$\mathrm{Cu} / \mathrm{ZnO}$-based catalysts have long been used for a variety of industrially and environmentally important processes, including methanol synthesis, ${ }^{\mathbf{1 - 1 0}}$ methanol steam reforming, ${ }^{\mathbf{1 1}, \mathbf{1 2}}$ and the water-gas shift reaction; ${ }^{\mathbf{1 3} 14}$ yet despite the extensive use of these catalysts, much remains to be fully understood in terms of the role of $\mathrm{ZnO}$ in facilitating catalytic activity and the effect of $\mathrm{ZnO}$ surface structure in determining $\mathrm{Cu}$ cluster growth. The dominant facets of wurzite $\mathrm{ZnO}$ are the non-polar (10 $\overline{1} 0)$ and $(11 \overline{2} 0)$ surfaces, and the polar (0001) and $(000 \overline{1})$ facets; clearly, the different surface structures presented

${ }^{a}$ Research Complex at Harwell, Rutherford Appleton Laboratory, Harwell Oxford, Didcot, Oxon, OX11 OFA, UK. E-mail: highamm@cardiff.ac.uk

${ }^{b}$ Cardiff University, School of Chemistry, Main Building, Park Place, Cardiff, CF10 3AT, UK

${ }^{c}$ Department of Physics and Astronomy, University College London, Gower Street, London WC1E 6BT, UK

${ }^{d}$ Kathleen Lonsdale Materials Chemistry, Department of Chemistry, University College London, 20 Gordon Street, London WC1H OAJ, UK

$\dagger$ Electronic supplementary information (ESI) available. See DOI: 10.1039/d0ta08351h by these four terminations will have consequences for $\mathrm{Cu}$ cluster growth. However, for the polar (0001) and (0001) surfaces, their complexity is further enhanced by their atomic reconstruction, which is essential for the stability of these polar surfaces. Atomic reconstruction in polar surfaces can significantly stabilise them to the extent that their stability is able to compete with non-polar surfaces. ${ }^{15}$ Moreover, it has been established that high levels of disorder in $\mathrm{ZnO}$ polar surfaces result in a significant configurational entropy contribution, enhancing stabilisation. ${ }^{16}$ Experimentally, studies have demonstrated that the polar ZnO surfaces are highly active in hydrogenation processes such as methanol synthesis. ${ }^{17,18}$ In particular, ZnO polar surfaces have been shown to play a key role in the synthesis of methanol.

STM studies reported that different $\mathrm{Cu}$ cluster growth behaviours were observed for the $\mathrm{Zn}$ - and O-terminated surfaces. ${ }^{19,20}$ Three-dimensional $\mathrm{Cu}$ cluster growth and surface pits with O-terminated edges, which have been suggested to act as $\mathrm{Cu}$ nucleation sites, were observed on the Zn-terminated surface. ${ }^{20}$ In contrast, for the O-terminated surface, 2-D Cu clusters were observed, although a more detailed analysis was limited by the limitations of the STM on O-teminated surfaces. ${ }^{19}$ 
A wide variety of $\mathrm{ZnO}$ surface features are likely to be present between different samples, thus allowing adsorbed $\mathrm{Cu}$ clusters to show different morphologies.

$\mathrm{Cu}$ cluster growth over the non-polar $\mathrm{ZnO}$ (1010) surface has already been subject to rigorous computational investigation, with studies utilising an unbiased Monte Carlo exploration of the energy landscape for $\mathrm{Cu}$ clusters supported on $\mathrm{ZnO}(10 \overline{1} 0)$ surfaces using interatomic potentials (IP) techniques, to identify accurately global minimum energy structures, which were then subsequently optimised using a more accurate plane-wave DFT approach. ${ }^{21}$ The studies revealed significantly wetting of $\mathrm{Cu}$ clusters when adsorbed on $\mathrm{ZnO}(10 \overline{1} 0)$, with supported clusters having considerably different structures compared to the gasphase $\mathrm{Cu}$ clusters. This result was attributed to a strong $\mathrm{Cu}-$ Zn interaction, in line with observations made in past experimental work. More recently, Cu cluster growth on ZnO (1010) surfaces was investigated using global optimisation techniques employing genetic algorithms in combination with highdimensional neural network potentials; ${ }^{22}$ the results of this approach suggest that small $\mathrm{Cu}$ clusters tend to form 3-D clusters, in contrast to the previous studies. It was also reported that at the metal-oxide interface, facets resembling the lowindex $\mathrm{Cu}(111)$ and $\mathrm{Cu}(110)$ surfaces emerge, which could be important in facilitating catalytic activity for $\mathrm{CO}_{2}$ conversion to methanol. These computational studies reflects the utility of IP techniques coupled with an unbiased global optimisation approach as an effective screening method for exploring a vast array of surface structures, with a generally good agreement in terms of geometric structure and relative stability of competing stable minimum structures between IP and DFT optimised results, allowing for a rigorous investigation of surface structures.

In the light of the development of this new approach to computational exploration of surface structures, a similar method was used to rationalise reconstruction of the polar ZnO surface which has already been identified as an important phenomenon in surface stability. ${ }^{15}$ The studies examined different surface reconstructions by considering a range of different surface layer occupancies, transferring $m \mathrm{Zn}$ or $\mathrm{O}$ atoms from the $\mathrm{Zn}$ - or O-terminated surfaces, to the other side of the slab (O- or Zn-terminated surfaces, respectively), resulting in pairs of surfaces featuring surface vacancies on one side, and surface adatoms on the other. The studies revealed an optimal value $m=6$, in order to minimise effectively the slab dipole moment with the $(5 \times 5)$ supercell used, and thus stabilise the polar $\mathrm{ZnO}$ surface. Applying the grand canonical ensemble method to determine the optimal surface occupancy revealed a drive towards full surface occupancy under typical conditions for both the $\mathrm{Zn}$ - and O-terminated reconstructed surfaces. Hence, it is expected that both surfaces consist of essentially full surface layer occupancy, with $6 \mathrm{Zn}(\mathrm{O})$ vacancies or $6 \mathrm{O}(\mathrm{Zn})$ adatoms for the $\mathrm{Zn}$ terminated (O-terminated) surface, to eliminate the surface dipole within the $(5 \times 5)$ supercell used. These detailed computational investigations provide an ideal starting point for consideration of $\mathrm{Cu}$ cluster growth on realistic model reconstructed polar ZnO surfaces (Fig. 1).
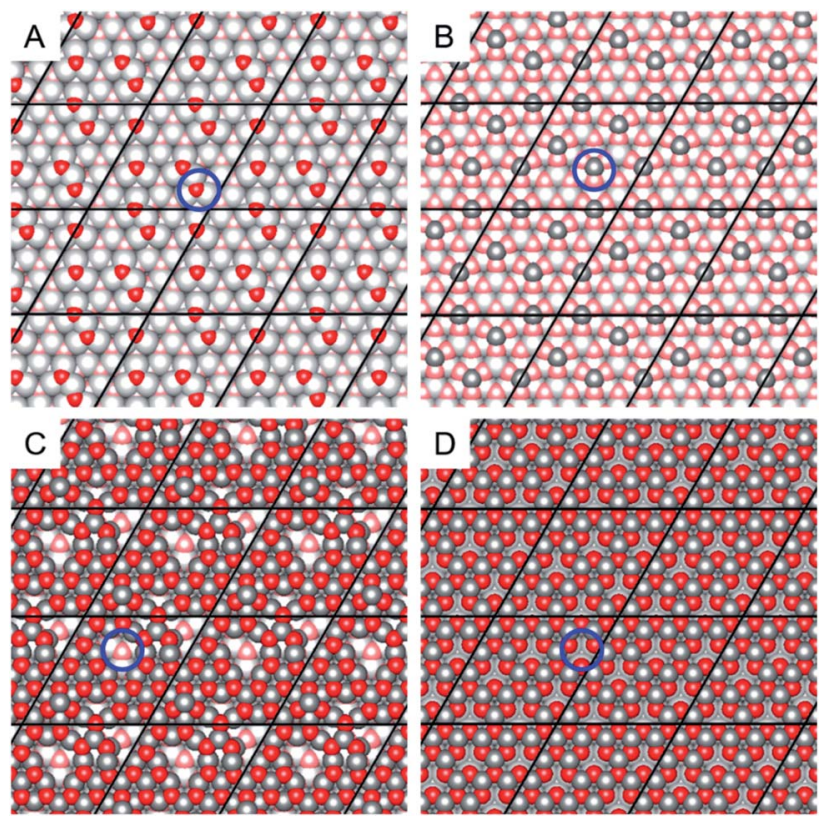

Fig. 1 The four different reconstructed $\mathrm{ZnO}$ surfaces considered in the present work, identified as representative surfaces by Mora-Fonz et al. ${ }^{15}$ The left panels ( $A$ and $C$ ) show the $\mathrm{Zn}$-terminated surface, whilst the right panels $(B$ and $D$ ) show the O-terminated surface. The top panels ( $A$ and $B$ ) depict reconstructions featuring $O(Z n)$ adatoms atop a complete $\mathrm{ZnO}$ layer, whilst the bottom panels ( $C$ and $D)$ show complete $\mathrm{ZnO}$ surface layers featuring $\mathrm{Zn}(\mathrm{O})$ vacancies. Grey spheres correspond to $\mathrm{Zn}$ atoms, whilst red spheres correspond to $\mathrm{O}$ atoms; atoms in the topmost layer are in bold colours whilst subsurface atoms are in faded colours. The blue circles and labels indicate the topmost surface features, either $\mathrm{Zn}$ or $\mathrm{O}$ adatoms or vacancies originating from the surface reconstruction. The graphics above show the extended periodic structure of the surface with the $(5 \times 5)$ surface supercells used in the calculations indicated by black lines.

The polar $\mathrm{ZnO}(0001)$ and (0001) facets have already been subject to computational investigation using hybrid QM/MM methods, ${ }^{23-28}$ investigating the possible participation of the $\mathrm{ZnO}$ surfaces in $\mathrm{CO}_{2}$ activation for methanol synthesis over the unreconstructed O-terminated surface ${ }^{23}$ and model reconstructed surfaces for both the $\mathrm{Zn}$ - and O-terminated facets, ${ }^{25}$ as well as for $\mathrm{Cu}$ cluster growth. The studies identify vacant $\mathrm{O}$ interstitial surface sites as being key to $\mathrm{CO}_{2}$ hydrogenation activity over the O-terminated $\mathrm{ZnO}$ surface, whilst it is suggested that $\mathrm{Cu}$ cluster growth is dependent on the presence of oxidised $\mathrm{Cu}^{+}$and $\mathrm{Cu}^{2+}$ to serve as anchors for cluster growth. Whilst these hybrid QM/MM studies provide many useful insights into the nature of the polar $\mathrm{ZnO}$ surface, the manner in which the reconstruction of the surface is modelled has its limitations, with surface vacancies introduced into the embedded cluster model not accounting for the density and distribution of surface vacancies. ${ }^{15}$ Hence, it is of interest to consider the nature of $\mathrm{Cu}$ cluster growth over these latest models for $\mathrm{ZnO}$ surface reconstruction.

In previous DFT studies investigating $\mathrm{CO}_{2}$ activation over a model catalyst consisting of a $\mathrm{Cu}_{8}$ cluster supported on a $\mathrm{ZnO}$ $(000 \overline{1})$ surface ${ }^{29}$ it was revealed that the structural flexibility of the $\mathrm{Cu}$ nanoparticle plays a key role in the extent of $\mathrm{CO}_{2}$ 
activation. Additionally, experimental studies investigating the catalytic activity of $\mathrm{Cu}$ clusters on an $\mathrm{Al}_{2} \mathrm{O}_{3}$ support show that catalytic activity is correlated with $\mathrm{Cu}$ cluster size. ${ }^{30}$ Experimental investigations of the $\mathrm{CO}_{2}$ hydrogenation activity of $\mathrm{ZnO}$ nanorod-supported $\mathrm{Cu}$ clusters suggest that the surface morphology of the $\mathrm{ZnO}$ nanorods greatly influence $\mathrm{Cu}$ cluster growth and have a critical impact on $\mathrm{CO}_{2}$ hydrogenation activity. ${ }^{31}$ Hence, it is of interest to determine precisely how reconstruction of the $\mathrm{ZnO}$ surface affects $\mathrm{Cu}$ cluster size morphology, and in turn, $\mathrm{CO}_{2}$ adsorption and related catalytic activity.

In this work, the $\mathrm{Cu} / \mathrm{ZnO}$ potential energy landscape was explored using IP MC techniques, from which low energy structures were refined with a DFT approach. Four distinct reconstructed $\mathrm{ZnO}$ surface structures were selected, representing O- (Zn-) rich $\mathrm{Zn}$ - (O-) terminated surfaces and $\mathrm{O}-(\mathrm{Zn}-)$ poor $\mathrm{O}-(\mathrm{Zn}-)$ terminated surfaces, as they were determined to be the most stable under typical conditions as revealed by the grand canonical ensemble studies performed previously. ${ }^{15}$ The role of surface features arising from reconstruction of the polar $\mathrm{ZnO}$ surface in determining the shape of $\mathrm{Cu}$ clusters is investigated. Moreover, we provide model $\mathrm{Cu} / \mathrm{ZnO}$ catalyst structures for future studies investigating the mechanism for $\mathrm{CO}_{2}$ hydrogenation to methanol.

\section{Methods and computational details}

This work uses a similar MC approach to that established by previous studies ${ }^{\mathbf{1 5 , 2 1}}$ for filtering low energy $\mathrm{Cu} / \mathrm{ZnO}$ structures with interatomic potentials (IP) and a subsequent DFT refinement using a plane-wave DFT approach. Details of the potential energy exploration is given below.

\section{Global optimisation of $\mathrm{Cu} / \mathrm{ZnO}$ structures}

We use the Knowledge-Led Master Code (KLMC) ${ }^{32-34}$ to perform an unbiased Monte Carlo (MC) exploration of the energy landscape for $\mathrm{Cu}_{n}$ clusters (for $n=1-8,24,50$ ) on four reconstructed polar ZnO (0001) and (0001) facets obtained by Mora-Fonz et $a{ }^{15}$ These four surface structures were selected as they were determined to be the most stable under typical conditions as revealed by the grand canonical ensemble studies performed previously. ${ }^{15}$ The MC routine generated 10000 initial random $\mathrm{Cu}_{n} / \mathrm{ZnO}$ structures by swapping $\mathrm{Cu}$ atoms over a lattice mesh placed atop the $\mathrm{ZnO}$ surfaces. The $\mathrm{ZnO}$ slabs consisted of a 6layer $(5 \times 5)$ supercell model, as described previously, ${ }^{15}$ whilst the $\mathrm{Cu}$ mesh consisted of 3-layer $(7 \times 7) \mathrm{Cu}(111)$ slab, for a total of 147 possible $\mathrm{Cu}$ sites. The $(7 \times 7) \mathrm{Cu}(111)$ slab was used as this afforded the best match to the ZnO slab dimensions based on the DFT optimised bulk $\mathrm{Cu}$ lattice parameter. The $\mathrm{Cu}$ mesh was placed $2.5 \AA$ above the topmost atoms of the ZnO slab.

\section{Interatomic potentials calculations}

Optimisation of the KLMC-generated structures was performed using the Generalised Utility Lattice Programme (GULP), ${ }^{35,36}$ applying a polarisable shell model potential to the $\mathrm{ZnO}$ oxygen atoms. $^{37}$ The $\mathrm{Cu}-\mathrm{ZnO}$ potentials used were derived by Mora-
Fonz et al., ${ }^{21}$ with Buckingham and Morse potentials describing the $\mathrm{Cu}-\mathrm{O}$ and $\mathrm{Cu}-\mathrm{Zn}$ interactions, respectively, whilst $\mathrm{Cu}-\mathrm{Cu}$ interactions were accounted for using many-body Gupta potentials. ${ }^{38}$ All of the $\mathrm{Cu}$ atoms were fully relaxed, as were the top three $\mathrm{ZnO}$ layers. Following the established procedure for modelling the reconstructed $\mathrm{ZnO}$ surfaces, ${ }^{15}$ a $2 \mathrm{D}$ periodic surface model was used, with uniform charge compensation applied to the bottom of the slab to account for the charge mismatch inherent in the approach employed to model the ZnO surface reconstruction, and a mesh of 100 fixed point charges at a distance of $50 \AA$ above and below the $\mathrm{Cu} / \mathrm{ZnO}$ slab was included to eliminate the residual dipole moment. Structural optimisation within GULP was performed using the BGFS algorithm; the lowest five structures obtained were identified for each $\mathrm{Cu}_{n}$ cluster and their relative stabilities compared. Structures for the five lowest energy configurations are freely and publicly available via the SAINT database (https:// saint.chem.ucl.ac.uk/).

\section{Periodic ab initio calculations}

To verify the KLMC-GULP approach, the five lowest energy $\mathrm{Cu}_{8}$ structures were refined using DFT methods; the DFT optimised structures are also available via the SAINT database. Refinement of the IP-optimised structures was performed using plane-wave DFT as implemented in the Vienna $A b$ initio Simulation Package (VASP). ${ }^{39,40}$ Interactions between core and valence electrons were described using the Projector Augmented Wave (PAW) pseudopotential approach. ${ }^{\mathbf{4 1 , 4 2}}$ The PBESol exchange correlation functional was used throughout, ${ }^{43}$ as was a plane-wave cut-off energy of $450 \mathrm{eV}$, which was deemed to be sufficient to obtain accurate DFT optimised geometries. All calculations were performed using $\Gamma$-centred $k$-mesh of $(1 \times 1 \times 1)$. As for the IP optimisations, all $\mathrm{Cu}$ atoms were allowed to relax, as were the top three $\mathrm{ZnO}$ layers, until the forces on the atoms were converged to within $0.01 \mathrm{eV} \AA^{-1}$. As a 3D periodic model was used, a vacuum gap of $18 \AA$ was introduced between the top $\mathrm{ZnO}$ layer of the slab and the bottom layer of the adjacent periodic image. For each of the $\mathrm{ZnO}$ slabs, $\mathrm{Zn}$ or $\mathrm{O}$ atoms were removed randomly from the bottom of the slab to eliminate any charge mismatch arising from the reconstruction (rather than applying a uniform charge compensation as was done for the IP calculations); likewise, the fixed-point charges used in the IP calculations to eliminate the residual surface dipole moment were replaced by a dipole correction as implemented in VASP.

\section{Results}

\section{Relative stability of minimum energy IP structures and $\mathrm{Cu}$ cluster adsorption energies}

Zn-rich O-terminated surface. The structures of the lowest $\mathrm{Cu} / \mathrm{ZnO}(000-1)$ energy structures are shown in Table 1 , whilst the corresponding relative energies for the five lowest energy structures for each $\mathrm{Cu}$ cluster size are presented in Table 2; higher energy structures can be found in the ESI (Table S1 $\dagger$ ). This reconstruction features a total of $6 \mathrm{Zn}$ adatoms per $(5 \times 5)$ surface supercell (hence we will describe this surface as a "Zn-rich" O- 
Table 1 Graphics for lowest energy structures obtained for adsorbed Cu clusters of various sizes. See ESI for the remaining 4 candidate local minimum structures (Table S1). Blue spheres represent $\mathrm{Cu}$, whilst red and grey spheres represent $\mathrm{O}$ and $\mathrm{Zn}$, respectively. The top ZnO layer (i.e. involved in the reconstruction) atoms are highlighted with darker spheres, whilst the subsurface $\mathrm{ZnO}$ atoms are represented by faded spheres. Thin green lines indicate cell boundaries. IP-calculated adsorption energies with respect to bulk Cu are listed below the graphics for each lowest energy structure

1

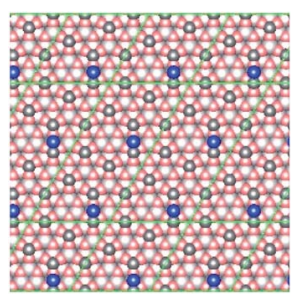

2.552

6

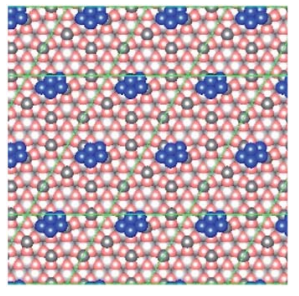

0.494
2

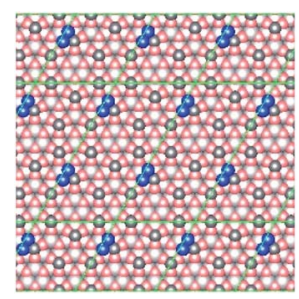

1.194
No. $\mathrm{Cu}$

3

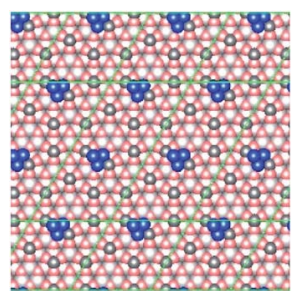

4

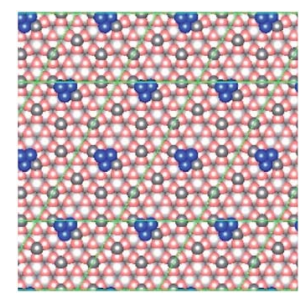

0.574

24
5

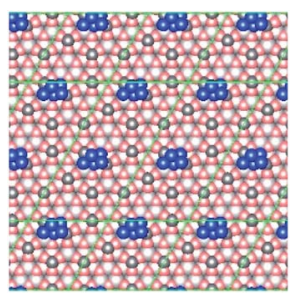

0.535
No. Cu

8

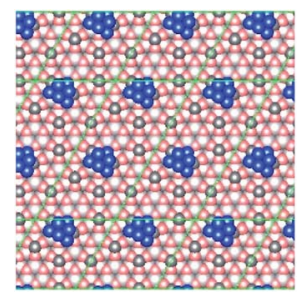

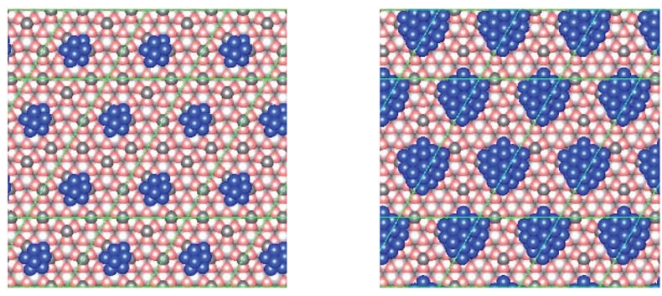

0.219

Adsorption energy w.r.t. bulk $\mathrm{Cu} / \mathrm{eV}$

0.391
50

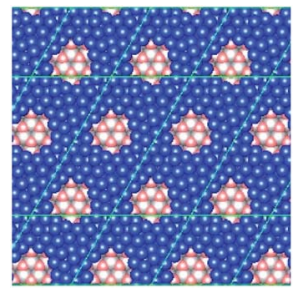

0.102

Table 2 Relative energies (in eV) for the 5 lowest energy structures for adsorbed Cu clusters of various sizes. The tabulated values are calculated with respect to the global minimum energy structure. See ESI for corresponding structures (Table S1)

\begin{tabular}{|c|c|c|c|c|c|c|c|c|c|c|}
\hline \multicolumn{11}{|c|}{ No. $\mathrm{Cu}$} \\
\hline Rank & 1 & 2 & 3 & 4 & 5 & 6 & 7 & 8 & 24 & 50 \\
\hline 2 & 0.059 & 0.009 & 0.028 & 0.446 & 0.060 & 0.070 & 0.068 & 0.074 & 0.079 & 0.084 \\
\hline 3 & 0.073 & 0.012 & 0.032 & 0.454 & 0.115 & 0.135 & 0.140 & 0.309 & 0.222 & 0.299 \\
\hline 4 & 0.077 & 0.072 & 0.045 & 0.546 & 0.130 & 0.165 & 0.203 & 0.358 & 0.243 & 0.302 \\
\hline
\end{tabular}

terminated surface), which are important in $\mathrm{Cu}$ cluster growth. For up to $3 \mathrm{Cu}$ atoms, we see that the structures are all very close in energy, with the $1^{\text {st }}$ and $5^{\text {th }}$ ranked structures being within less than $\sim 0.1 \mathrm{eV}$ of each other. This result can be attributed to the fact that there is no significant variation in the structure of the $\mathrm{Cu}$ "cluster", hence the different structures simply represent different adsorption sites on the reconstructed $\mathrm{ZnO}$ surface. Indeed, as can be seen from the graphics for the competing structures presented in the ESI (Table S1 $\dagger$ ), all the structures feature almost identical groups of $\mathrm{Cu}$ atoms adjacent to a surface $\mathrm{Zn}$ adatom, merely in a variety of different orientations.

However, for $n>4$, a greater variation is seen between the IP lowest minimum energy structure and the competing local minima, which is especially pronounced for the $\mathrm{Cu}_{4}$ cluster, whose lowest minimum energy structure is found to be remarkably stable, by over $0.44 \mathrm{eV}$, compared to the second most stable local minimum. Additionally, a preference for 3dimensional $\mathrm{Cu}$ cluster growth emerges; the top 3 most stable structures feature a highly coordinated tetrahedral $\mathrm{Cu}$ cluster, whereas the local minimum structures ranked $4^{\text {th }}$ and $5^{\text {th }}$ exhibit a planar $\mathrm{Cu}$ cluster geometry, with the $\mathrm{Cu}$ plane lying incommensurate with plane of the topmost complete $\mathrm{ZnO}$ surface layer, coordinating with a surface $\mathrm{Zn}$ adatom. The remarkable stability of the $\mathrm{Cu}_{4}$ global minimum structure can be attributed to its optimal distance between the $\mathrm{Cu}$ cluster and adjacent $\mathrm{Zn}$ surface adatoms; in the global minimum energy structure, the $\mathrm{Cu}_{4}$ tetrahedron is located equidistantly between $\mathrm{Zn}$ surface adatom nearest neighbours, hence maximising the 
favourable attractive interaction between $\mathrm{Zn}$ and $\mathrm{Cu}$. For the other two local minima featuring $\mathrm{Cu}_{4}$ tetrahedra, the $\mathrm{Cu}_{4}$ cluster is adsorbed such than only one surface $\mathrm{Zn}$ adatom is well-coordinated with the $\mathrm{Cu}$ cluster.

For larger $\mathrm{Cu}$ clusters, the high stability of the $\mathrm{Cu}_{4}$ lowest minimum energy structure is reflected in the fact that because the most stable local minimum energy structures are based around the $\mathrm{Cu}_{4}$ global minimum energy structure, e.g. for the $\mathrm{Cu}_{5}$ clusters, the 5 top-ranked local minima are identical apart from the positioning of the $5^{\text {th }} \mathrm{Cu}$ atom, with each of the five clusters featuring the tetrahedral $\mathrm{Cu}_{4}$ arrangement and adsorption site between two surface $\mathrm{Zn}$ adatoms as observed for the $\mathrm{Cu}_{4}$ global minimum energy structure. Indeed, the global minimum structures up to $\mathrm{Cu}_{8}$ represent sequential growth, with each subsequent structure being virtually identical apart from the additional $\mathrm{Cu}$ atom. We also note that the top two most stable structures for $\mathrm{Cu}_{5}, \mathrm{Cu}_{6}, \mathrm{Cu}_{7}$ and $\mathrm{Cu}_{8}$ clusters feature very similar $\mathrm{Cu}$ cluster geometries adsorbed on the same $\mathrm{ZnO}$ surface site, and differ only in their rotational orientation, as is evident in the IP-calculated relative energies, which show that $3^{\text {rd }}, 4^{\text {th }}$ and $5^{\text {th }}$ ranked structures are all much closer in terms of relative energy to each other, and are further in terms of relative energy from the $1^{\text {st }}$ and $2^{\text {nd }}$ ranked structures. This sequence is especially evident for the $\mathrm{Cu}_{8}$ clusters, with the $1^{\text {st }}$ and $2^{\text {nd }}$ ranked structures being very close in relative stability (with 0.08 $\mathrm{eV}$ ), whilst the $3^{\text {rd }}, 4^{\text {th }}$ and $5^{\text {th }}$ ranked structures are all considerably less stable $(>0.22 \mathrm{eV}$ difference in relative stability between $2^{\text {nd }}$ and $3^{\text {rd }}$ ranked structures) and close to each other in relative stability (within $0.06 \mathrm{eV}$ ).

These trends identified in the relative stability are present for the larger $\mathrm{Cu}_{24}$ cluster, but less pronounced. It is notable that these much larger clusters tend to be positioned such that up to 4 surface $\mathrm{Zn}$ adatoms are occluded by the $\mathrm{Cu}$ cluster, rather than the $\mathrm{Cu}$ cluster being coordinated by only $2 \mathrm{Zn}$ adatoms as seen for the $\mathrm{Cu}_{4}-\mathrm{Cu}_{8}$ global minimum structures. This behaviour can simply be attributed to the larger cluster being more effectively able to maximise its favourable attractive interactions with the $\mathrm{Zn}$ adatoms at this adsorption site. We also see that the $\mathrm{Cu}_{24}$ cluster, whilst covering a significantly greater area of the $\mathrm{ZnO}$ surface, is not at an appreciably greater height than the smaller clusters; it can be seen in the side view presented in Fig. 2 that both the $\mathrm{Cu}_{8}$ and $\mathrm{Cu}_{24}$ global minimum supported $\mathrm{Cu}$ clusters consist of 2-3 $\mathrm{Cu}$ layers, with the topmost $\mathrm{Cu}$ atoms in the $\mathrm{Cu}_{24}$ cluster lying no more than $4.17 \AA$ above the topmost subsurface atoms (i.e. not the $\mathrm{Zn}$ surface adatom layer); the corresponding distance for the $\mathrm{Cu}_{8}$ and $\mathrm{Cu}_{1}$ global minima were $3.04 \AA$ and $1.72 \AA$, respectively. It is also worth noting that for the $\mathrm{Cu}_{24}$ global minimum structure, the topmost subsurface atoms were lattice $\mathrm{Zn}$, whilst for the smaller $\mathrm{Cu}_{8}$ cluster, the topmost subsurface atoms were lattice $O$, suggesting that the strong $\mathrm{Cu} / \mathrm{Zn}$ interaction lifts the subsurface $\mathrm{Zn}$ above the subsurface $\mathrm{O}$ for the larger cluster. Additionally, comparing the top 5 most stable local minimum energy structures, whilst 4 of the 5 minima correspond to discrete, isolated $\mathrm{Cu}$ clusters, coalescence of the $\mathrm{Cu}$ clusters can be seen in the $4^{\text {th }}$ ranked structure. It is likely that this is at least in part an artefact of the limited size of the $\mathrm{ZnO}$ surface supercell that can be used, as

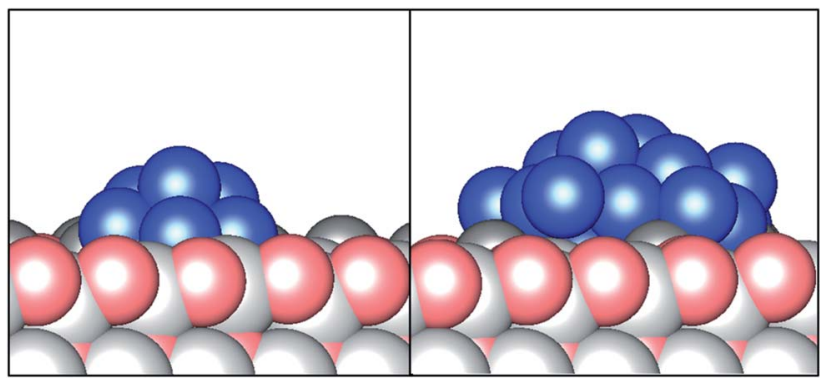

Fig. 2 Side view of the global minimum energy structures for $\mathrm{Cu}_{8}$ (left) and $\mathrm{Cu}_{24}$ (right) clusters adsorbed on the reconstructed $\mathrm{O}$-terminated $\mathrm{ZnO}$ surface featuring $\mathrm{Zn}$ adatoms.

identified in the previous studies on the $\mathrm{ZnO}$ reconstruction. ${ }^{\mathbf{1 5}}$ Whilst the $(5 \times 5)$ supercell is largely sufficient to allow for near complete elimination of the slab dipole moment through surface reconstruction, it cannot be excluded that a larger supercell would enable a greater variety of structures to be sampled; such a possibility is, however, largely outside the realm of computational exploration using present methods and resources. Likewise, the size of the $(5 \times 5)$ supercell imposes a limit on the size of discrete $\mathrm{Cu}$ clusters that can be investigated in this study.

For very high $\mathrm{Cu}$ loading $(50 \mathrm{Cu}$ per $\mathrm{ZnO}(5 \times 5)$ supercell), the $\mathrm{ZnO}$ surface is saturated with $\mathrm{Cu}$, achieving approximately 2 monolayer coverage. Nonetheless, the structures obtained in this study provide insights into the nature of the interaction of $\mathrm{Cu}$ with the $\mathrm{ZnO}$ polar surface. For all of the top ranked 5 local minima, there is no apparent $\mathrm{Cu}$ coverage in the hexagonal region - which represents the greatest expanse of the slab surface uninterrupted by surface $\mathrm{Zn}$ adatoms - of the reconstructed ZnO surface. This observation is consistent with the fact that the $\mathrm{Cu}-\mathrm{Zn}$ interaction is attractive, as shown by the IP terms. It was also found that height of the topmost $\mathrm{Cu}_{50}$ atom is slightly less than that of the $\mathrm{Cu}_{24}$ cluster, relative to the topmost ZnO subsurface atoms. This observation would suggest that for a given number of $\mathrm{Cu}$ atoms, upward $\mathrm{Cu}$ cluster growth becomes less favourable than outward wetting of the Cu cluster, with the further addition of $\mathrm{Cu}$ resulting in spreading of the cluster to interact with additional nearby surface $\mathrm{Zn}$ adatoms.

The presence of $\mathrm{Zn}$ adatoms plays, therefore, a key role in $\mathrm{Cu}$ cluster growth. Moreover, this surface feature appears to support the growth of 3D Cu clusters, rather than flat, planar $\mathrm{Cu}$ arrays on the $\mathrm{ZnO}$ surface, with the $\mathrm{Cu}$ clustering around the $\mathrm{Zn}$ adatoms and the growth of $\mathrm{Cu}$ clusters being most favourable at surface sites that maximise contact between the $\mathrm{Cu}$ cluster and the $\mathrm{Zn}$ adatoms. Whilst an attractive interaction between $\mathrm{Cu}$ and $\mathrm{Zn}$ had been reported previously in experimental ${ }^{\mathbf{1 9 , 2 0}}$ studies and in computational studies of the non-polar surface using a similar methodology to the present work, ${ }^{21}$ previous work suggested planar $\mathrm{Cu}$ cluster formation, for both the non-polar $\mathrm{ZnO}$ surface and for O-terminated surfaces. The 3D cluster formation reported here provides further evidence that the features of the reconstructed polar $\mathrm{ZnO}$ surface determine adsorbed $\mathrm{Cu}$ cluster morphology. 
Adsorption energies with respect to bulk $\mathrm{Cu}$ as calculated using the same many-body $\mathrm{Cu}-\mathrm{Cu}$ potentials are also presented in Table 1, according to the equation $E_{\text {ads }}=E_{\mathrm{Cu}_{n} / \mathrm{ZnO}}-$ $E_{\mathrm{ZnO}}-n E_{\mathrm{Cu}}$, where the adsorption energy is $E_{\text {ads }}, n$ is the number of $\mathrm{Cu}$ atoms in the cluster, the energy for the $\mathrm{Cu}_{n}$ cluster adsorbed on the reconstructed $\mathrm{ZnO}$ surface is $E_{\mathrm{Cu}_{n} / \mathrm{ZnO}}$, $E_{\mathrm{Zno}}$ is the total energy for the clean reconstructed $\mathrm{ZnO}$ surface, and $E_{\mathrm{Cu}}$ is the total bulk energy per $\mathrm{Cu}$ atom. We find that the $\mathrm{Cu}$ cluster adsorption becomes more exothermic for increasing numbers of $\mathrm{Cu}$ atoms present on the surface (Table 1), reflecting the stabilisation afforded by $\mathrm{Cu}-\mathrm{Cu}$ interactions and the overall increased stability of larger clusters, in agreement with the previous computational studies of the non-polar ZnO surface. ${ }^{21}$ However, it can also be seen that the calculated adsorption energy remains endothermic with respect to bulk $\mathrm{Cu}$ across the whole range of $\mathrm{Cu}$ cluster sizes.

O-poor O-terminated surface. We now consider the (000-1) $\mathrm{ZnO}$ surface featuring $6 \mathrm{O}$ vacancies in the surface layer (hence this surface is referred to as the "O-poor" O-terminated surface). The results are presented in Tables 3 and 4; likewise, graphics for the five lowest energy structures for each $\mathrm{Cu}$ cluster size can be found in the ESI (Table S2 $\dagger$ ).

In the three most stable $\mathrm{Cu}_{1} / \mathrm{ZnO}$ structures, we see that there are two main surface pits which serve to accommodate the $\mathrm{Cu}$ clusters. The $4^{\text {th }}$ and $5^{\text {th }}$ ranked structures, on the other hand, feature the $\mathrm{Cu}$ atom in smaller O-vacancy sites. The top three structures are all close in energy, within $0.05 \mathrm{eV}$, whereas

Table 3 Graphics for lowest energy structures obtained for adsorbed Cu clusters of various sizes. See ESI for the remaining 4 candidate local minimum structures (Table S2). Blue spheres represent $\mathrm{Cu}$, whilst red and grey spheres represent $\mathrm{O}$ and $\mathrm{Zn}$, respectively. The top ZnO layer (i.e. involved in the reconstruction) atoms are highlighted with darker spheres, whilst the subsurface $\mathrm{ZnO}$ atoms are represented by faded spheres. Thin green lines indicate cell boundaries. IP-calculated adsorption energies with respect to bulk Cu are listed below the graphics for each lowest energy structure

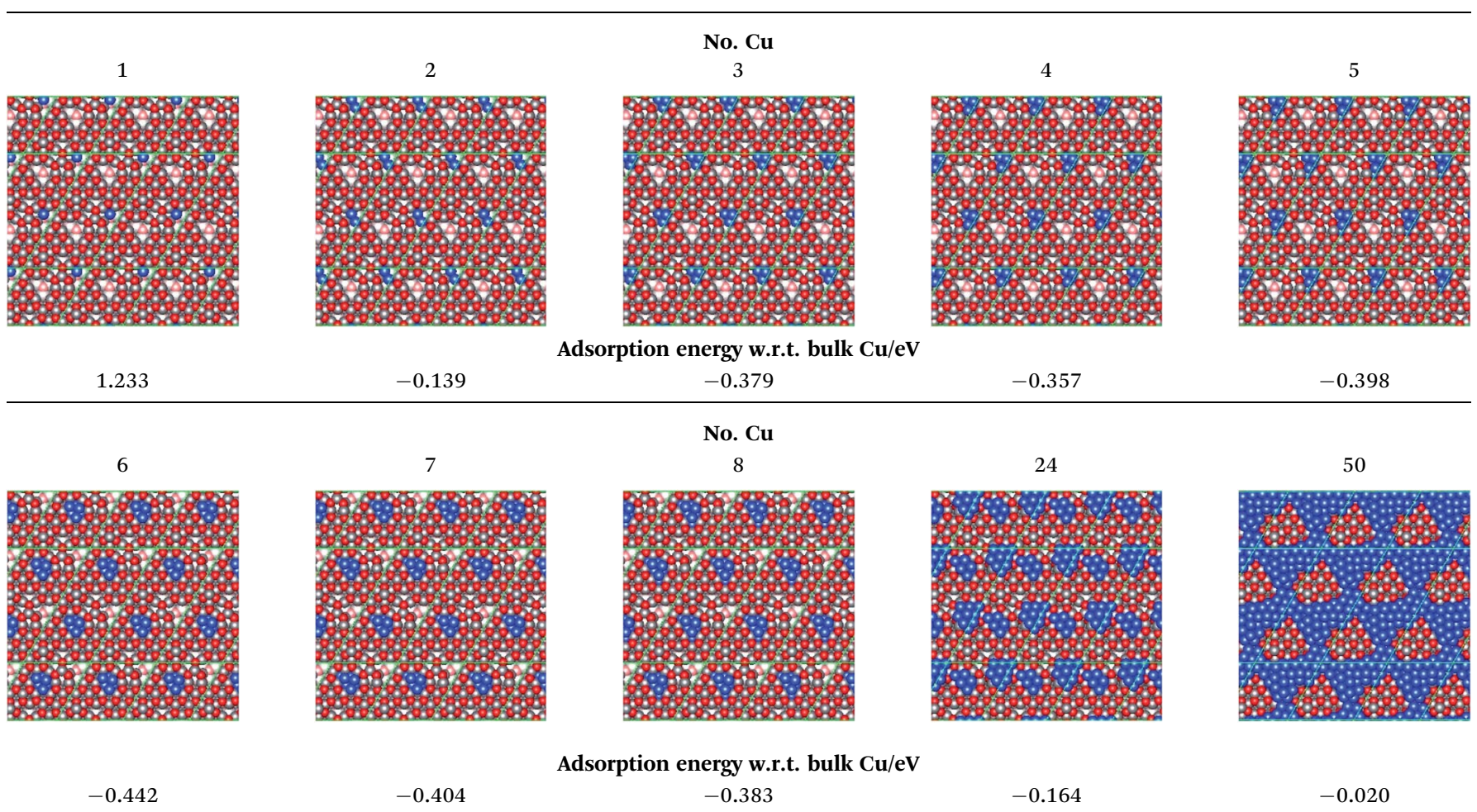

Table 4 Relative energies (in eV) for the 5 lowest energy structures for adsorbed Cu clusters of various sizes. The tabulated values are calculated with respect to the global minimum energy structure. See ESI for corresponding structures (Table S2)

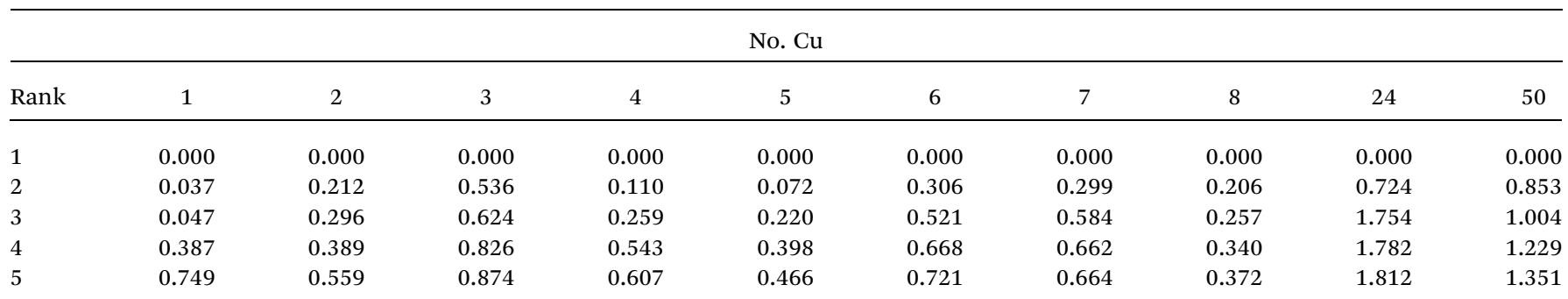


the $4^{\text {th }}$ and $5^{\text {th }}$ ranked structures are considerably less stable, at $>0.38 \mathrm{eV}$ less stable that the lowest minimum, which can be attributed to the higher number of $\mathrm{Cu}-\mathrm{Zn}$ interactions.

For smaller $\mathrm{Cu}$ clusters, up to $\mathrm{Cu}_{5}$, the lowest minimum structures feature a Cu cluster accommodated in the smaller of the two pits, whilst for $\mathrm{Cu}_{6}, \mathrm{Cu}_{7}$ and $\mathrm{Cu}_{8}$ lowest minima, the $\mathrm{Cu}$ clusters are located in the largest surface indentation because of the limited surface pit size. Structures with the $\mathrm{Cu}$ atom in the larger surface pit are present amongst the top 5 ranked structures for all $\mathrm{Cu}$ cluster sizes (see $\mathrm{ESI}^{\dagger}$ ). For larger $n$, however, $\mathrm{Cu}$ sits in both the smaller and larger surface pits with energy differences being within $\sim 0.3 \mathrm{eV}$. $\mathrm{Cu}$ clusters adsorbed in the smaller pit persist for $\mathrm{Cu}_{7}$ but are considerably less stable ( $>0.55 \mathrm{eV}$ less stable than the lowest minimum), and are completely absent from the top 5 ranked local minima for $\mathrm{Cu}_{8}$.

For higher $\mathrm{Cu}$ loadings $\left(\mathrm{Cu}_{24}\right)$, the lowest minimum has two smaller separate clusters, consisting of a smaller, flatter $\mathrm{Cu}_{9}$ cluster in the larger surface pit, and a larger, taller $\mathrm{Cu}_{15}$ cluster in the smaller pit. The structure suggests that the larger (smaller) surface pit can accommodate up to 8 (5) $\mathrm{Cu}$ atoms; subsequent $\mathrm{Cu}$ cluster growth predominantly takes place atop $\mathrm{Cu}$ atoms in the smaller pit and is driven primarily by $\mathrm{Cu}-\mathrm{Cu}$ interactions, since the undercoordinated $\mathrm{Zn}$ is no longer accessible to $\mathrm{Cu}$ and $\mathrm{Cu}-\mathrm{O}$ interactions are repulsive. We also find that the other local minimum energy structures for $\mathrm{Cu}_{24}$ show single, contiguous $\mathrm{Cu}$ clusters, spreading across the two surface pits that serve as nucleation sites for $\mathrm{Cu}$ cluster growth.
For very high $\mathrm{Cu}$ loading $\left(\mathrm{Cu}_{50}\right)$, as with the $\mathrm{Zn}$-rich Oterminated reconstructed surface, we see that the $\mathrm{ZnO}$ surface is saturated with $\mathrm{Cu}$, with a few islands present on the surface devoid of $\mathrm{Cu}$ growth. Comparing the local minimum energy structures for the $\mathrm{Zn}$-rich and O-poor O-terminated surfaces, we see that the $\mathrm{Cu}_{50}$ structures for the O-poor surface leaves a greater area of the $\mathrm{ZnO}$ surface exposed and free of $\mathrm{Cu}$. We can attribute this behaviour to the initial $\mathrm{Cu}$ cluster growth being predominantly within the surface pits, and thus smaller clusters do not take up an appreciable proportion of the total slab surface area, since initial $\mathrm{Cu}$ cluster growth is essentially vertical; hence for very high $\mathrm{Cu}$ loading, where $\mathrm{Cu}$ cluster growth begins to spread beyond the confines of the surface pits, the area of the slab covered is less than that for the Zn-rich surface, for which smaller clusters are more spread out on the surface initially.

The IP adsorption energies with respect to bulk $\mathrm{Cu}$ are also presented in Table 3. The calculated adsorption energies, in contrast to the Zn-rich O-terminated surface, are generally exothermic, with only the system containing a single adsorbed $\mathrm{Cu}$ atoms being unstable with respect to the bulk. This is probably due to high energetic cost associated with separating a single atom, rather than a cluster of atoms, from the bulk structure. For smaller $\mathrm{Cu}$ clusters, there is a general trend of increasingly exothermic adsorption energies with respect to bulk $\mathrm{Cu}$ as the cluster size grows, although we note that there are maxima in adsorption energies for $\mathrm{Cu}_{3}$ and $\mathrm{Cu}_{6}$ clusters. It is notable that the $\mathrm{Cu}_{3}$ cluster represents the largest $\mathrm{Cu}$ cluster

Table 5 Graphics for lowest energy structures obtained for adsorbed Cu clusters of various sizes. See ESI for the remaining 4 candidate local minimum structures (Table S3). Blue spheres represent $\mathrm{Cu}$, whilst red and grey spheres represent $\mathrm{O}$ and Zn, respectively. The top ZnO layer (i.e. involved in the reconstruction) atoms are highlighted with darker spheres, whilst the subsurface $\mathrm{ZnO}$ atoms are represented by faded spheres

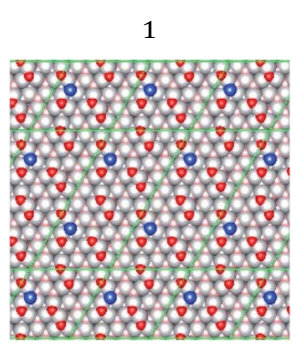

1.710

6

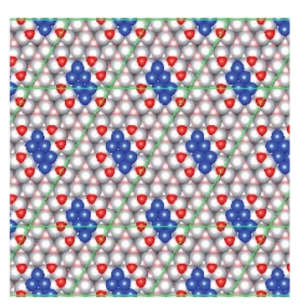

2

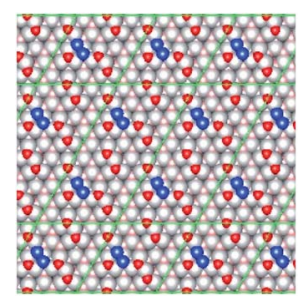

No. Cu

3

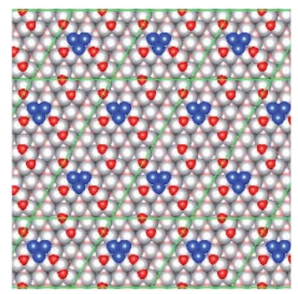

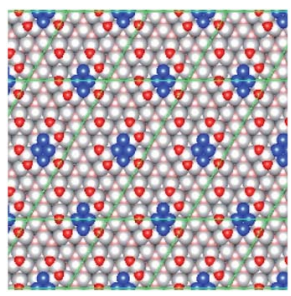

Adsorption energy w.r.t. bulk $\mathrm{Cu} / \mathrm{eV}$

0.414

0.084

$-0.031$

$-0.057$

No. $\mathrm{Cu}$

8

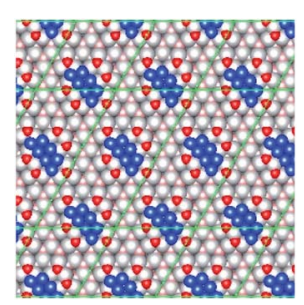

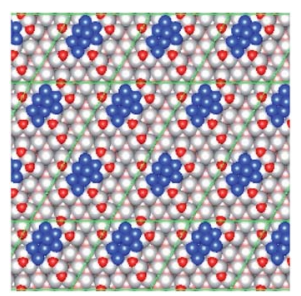

24

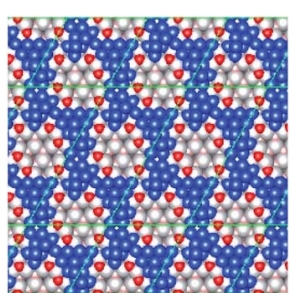

50

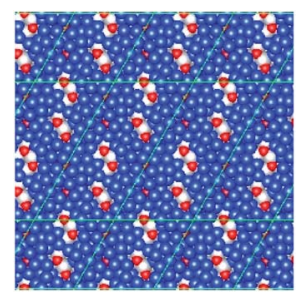

Adsorption energy w.r.t. bulk $\mathrm{Cu} / \mathrm{eV}$

$-0.065$

$-0.043$
$-0.040$
$-0.095$

$-0.086$ 
Table 6 Relative energies (in eV) for the 5 lowest energy structures for adsorbed Cu clusters of various sizes. The tabulated values are calculated with respect to the global minimum energy structure. See ESI for corresponding structures (Table S3)

\begin{tabular}{|c|c|c|c|c|c|c|c|c|c|c|}
\hline Rank & 1 & 2 & 3 & 4 & 5 & 6 & 7 & 8 & 24 & 50 \\
\hline 2 & 0.007 & 0.032 & 0.014 & 0.153 & 0.266 & 0.353 & 0.119 & 0.054 & 0.510 & 0.462 \\
\hline 3 & 0.012 & 0.190 & 0.188 & 0.243 & 0.422 & 0.396 & 0.245 & 0.113 & 0.552 & 0.493 \\
\hline 4 & 0.019 & 0.209 & 0.255 & 0.323 & 0.426 & 0.452 & 0.285 & 0.132 & 0.552 & 0.496 \\
\hline
\end{tabular}

size where the global minimum IP structure features the $\mathrm{Cu}$ cluster positioned within the smaller of the three O-vacancy surface pits; hence one can infer that the particular stability of the $\mathrm{Cu}_{3}$ and $\mathrm{Cu}_{6}$ clusters is related to the dimensions of the surface pits, which happen to be commensurate with the $\mathrm{Cu}$ clusters of these sizes to maximise attractive $\mathrm{Cu}-\mathrm{Cu}$ and $\mathrm{Cu}-\mathrm{Zn}$ interactions - with clusters consisting of greater numbers of $\mathrm{Cu}$ atoms being too large to accommodate optimally in these surface pits. This behaviour is further reflected in the considerably less exothermic adsorption energy relative to bulk $\mathrm{Cu}$ for the $\mathrm{Cu}_{24}$ and $\mathrm{Cu}_{50}$ systems, since clearly clusters of these sizes are far too large to fit within the $\mathrm{O}$ vacancy pits. Hence, the presence of these vacancy sites can be inferred as key to the stability of $\mathrm{Cu}$ clusters adsorbed on this particular surface reconstruction.

O-rich Zn-terminated surface. Turning now to the Znterminated surfaces, we will consider first the surface reconstruction featuring $\mathrm{O}$ adatoms distributed over the $\mathrm{ZnO}$ surface, (which we refer to as the "O-rich" Zn-terminated surface). The five lowest energy structures are presented in Table 5, with relative energies reported in Table 6; higher energy structures can be found in the ESI (Table S3†). Surface $\mathrm{O}$ adatoms form large hexagonal regions on the reconstructed surface (Fig. 3). For $\mathrm{Cu}_{1}$, we note that the local minimum energy structures are all very close in terms of energy (all within $0.025 \mathrm{eV}$ ), reflecting the fact that the favoured 3-coordinate hollow site between three surface $\mathrm{Zn}$ atoms is very similar for the different structures, with the small variations in stability being attributable to the marginal repulsive effect of nearby surface $\mathrm{O}$ adatoms. For $\mathrm{Cu}_{2}$ and $\mathrm{Cu}_{3}$ clusters, the range of stability is greater across the top 5 ranked local minimum energy structures, with all being within $\sim 0.3 \mathrm{eV}$, as the larger $\mathrm{Cu}$ cluster size limits the number of distinct surface sites that afford minimal repulsive interactions between $\mathrm{Cu}$ and $\mathrm{O}$ atoms; however, the observed structures reveal that there are still many surface sites that can accommodate clusters of up to $3 \mathrm{Cu}$ atoms. For larger clusters of 4 or $5 \mathrm{Cu}$ atoms, the lowest minima feature planar $\mathrm{Cu}$ clusters in the large hexagonal region enclosed by surface $\mathrm{O}$ adatoms, although planar $\mathrm{Cu}$ clusters outside this region still appear amongst the top 5 ranked structures. This behaviour can be attributed to the hexagonally enclosed region representing the greatest uninterrupted expanse of exposed surface $\mathrm{Zn}$ uninterrupted by surface $\mathrm{O}$ adatoms; hence larger (i.e., more than $3 \mathrm{Cu}$ atoms) are more easily accommodated within this region to maximise attractive $\mathrm{Cu}-\mathrm{Zn}$ interactions. Notably, in contrast to the two reconstructions of the O-terminated surface already discussed, for this $\mathrm{Zn}$-terminated surface, larger planar structures are favoured, whereas for the O-terminated surfaces, the lowest minima for $\mathrm{Cu}_{4}$ and larger clusters are invariably 3D structures, although a mixture of planar and 3D structures are observed amongst the top 5 ranked structures for all of the reconstructed $\mathrm{ZnO}$ surfaces discussed so far. The preference for planar $\mathrm{Cu}$ cluster growth on the O-rich Zn-terminated surface can be attributed simply to the fact that the unreconstructed surface presents essentially a close-packed sheet of surface $\mathrm{Zn}$ which necessarily would result in planar $\mathrm{Cu}$ cluster growth to maximise $\mathrm{Cu}-\mathrm{Zn}$ attractive interactions; in this particular reconstruction, the $\mathrm{O}$ adatoms merely serve to interrupt planar $\mathrm{Cu}$ cluster growth.

For intermediate $\mathrm{Cu}$ cluster sizes $\left(\mathrm{Cu}_{6}\right.$ and $\left.\mathrm{Cu}_{7}\right)$, the larger cluster size limits the number of surface sites that avoid repulsive $\mathrm{Cu}-\mathrm{O}$ interactions, with the structures featuring $\mathrm{Cu}$ clusters within the large hexagonal region being more stable. Examining the top 5 ranked local minimum structures for $\mathrm{Cu}_{6}$, the effects of the attractive $\mathrm{Cu}-\mathrm{Zn}$ interaction, and repulsive $\mathrm{Cu}-$

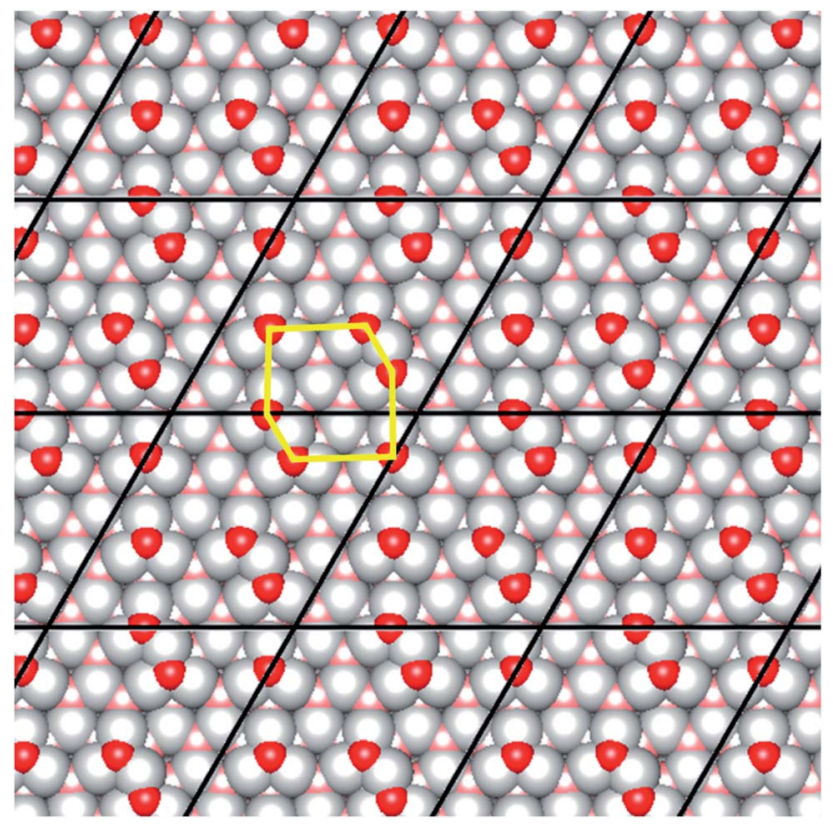

Fig. 3 Graphic illustrating the "hexagonal surface region" defined by surface $\mathrm{O}$ adatoms on the reconstructed $\mathrm{Zn}$-terminated $\mathrm{ZnO}$ surface; the region is defined by the yellow lines. 
$\mathrm{O}$ interaction, are especially pronounced; the global minimum energy structure, featuring a planar monolayer $\mathrm{Cu}_{6}$ cluster within the hexagonal region, is considerably more stable that the $2^{\text {nd }}$ ranked structure $(0.35 \mathrm{eV})$, which is also located within the hexagonal region, but rather adopts a 3D structure featuring a plane of $5 \mathrm{Cu}$ atoms in contact with the $\mathrm{ZnO}$ surface and a $6^{\text {th }}$ $\mathrm{Cu}$ atom located atop this $\mathrm{Cu}_{5}$ cluster. Hence, the difference in stability between the two structures can be largely attributed to the more strongly attractive $\mathrm{Cu}-\mathrm{Zn}$ interaction over the comparatively weaker $\mathrm{Cu}-\mathrm{Cu}$ interaction. Comparing the global minimum energy structure for the $\mathrm{Cu}_{6}$ cluster, with the $5^{\text {th }}$ ranked local minimum, illustrates well the impact of the repulsive interactions between $\mathrm{Cu}$ and $\mathrm{O}$; whilst the structure of the $\mathrm{Cu}$ clusters are identical, consisting of two rows of $3 \mathrm{Cu}$ atoms in a close-packed arrangement, the lowest minimum energy structure is more stable by $0.46 \mathrm{eV}$. Measuring the shortest distance between $\mathrm{Cu}$ and adatoms $\mathrm{O}$ reveals that in the global minimum structure, $\mathrm{Cu}$ atoms are separated from surface $\mathrm{O}$ adatoms by at least $3.1 \AA$; however, for the $5^{\text {th }}$ ranked local minimum structure, the minimum separation is only 3.0 Å. Simply put, for $\mathrm{Cu}$ clusters consisting of at least $6 \mathrm{Cu}$ atoms, the most stable planar configuration of $\mathrm{Cu}$ atoms is too large to be comfortably accommodated in the surface regions outside the hexagonal region defined by the surface $\mathrm{O}$ adatoms; hence the hexagonal region is the favoured site for adsorption of intermediate size planar $\mathrm{Cu}$ clusters.

For $\mathrm{Cu}_{8}$ clusters, a change in behaviour is observed as $3 \mathrm{D} \mathrm{Cu}$ cluster structures begin to emerge as the most favourable configuration, with the global minimum structure featuring a $\mathrm{Cu}_{7}$ planar cluster resembling that of the $4^{\text {th }}$ ranked local minimum structure for $\mathrm{Cu}_{7}$ clusters, with an additional $\mathrm{Cu}$ atom on top. Moreover, the global minimum energy structure is located outside the hexagonal region which was previously identified as being a favourable site for adsorption of smaller planar $\mathrm{Cu}$ clusters, whilst the remaining top candidate local minimum energy structures all feature 2D planar structures only, with the $2^{\text {nd }}, 3^{\text {rd }}$ and $4^{\text {th }}$ ranked structures being located within the hexagonal region. Additionally, the range of stabilities for the minimum energy structures is smaller than that obtained for smaller $\mathrm{Cu}_{6}$ and $\mathrm{Cu}_{7}$ clusters, with all structures being within $0.2 \mathrm{eV}$; only the local minima obtained for a single $\mathrm{Cu}$ atom are closer in energy than this. This result strongly suggests that the favourable hexagonal region can only comfortably accommodate up to $7 \mathrm{Cu}$ atoms in a planar arrangement, hence for larger clusters consisting of at least 8 $\mathrm{Cu}$ atoms, there is a degree of competition between larger planar clusters, which maximise the attractive $\mathrm{Cu}-\mathrm{Zn}$ interaction, although at the expense of increasing repulsive interactions between $\mathrm{Cu}$ and $\mathrm{O}$ adatoms, or alternatively the formation of 3D clusters, which avoid such repulsive interactions to a greater extent, but sacrifice attractive interactions between $\mathrm{Cu}$ and $\mathrm{Zn}$ in favour of the attractive $\mathrm{Cu}-\mathrm{Cu}$ interactions between the first and second layers of the 3D structure.

For larger $\mathrm{Cu}_{24}$ systems, we immediately note that the global minimum structure almost totally covers the $\mathrm{ZnO}$ surface outside the large hexagonal regions previously identified as being the ideal adsorption sites for intermediate sized $\mathrm{Cu}$ clusters. The remaining 4 top ranked local minimum energy structures are substantially similar, in all cases with the large hexagonal region devoid of $\mathrm{Cu}$ atoms, with only small variations in the connectivity between $\mathrm{Cu}$-covered areas on the remainder of the surface. These results suggest that for larger amounts of $\mathrm{Cu}$ deposited on the surface, which cannot possibly be accommodated in the distinctive hexagonal region, the remainder of the $\mathrm{Zn}$-terminated surface, outside the hexagonal region, is favoured for $\mathrm{Cu}$ adsorption, since it allows for improved $\mathrm{Cu}-\mathrm{Cu}$ interactions. Hence, it is evident that the relative importance of the three different kinds of interactions present (attractive $\mathrm{Cu}-$ $\mathrm{Zn}$, attractive $\mathrm{Cu}-\mathrm{Cu}$, and repulsive $\mathrm{Cu}-\mathrm{O}$ ) changes depending on the extent of surface loading of $\mathrm{Cu}$.

For very high $\mathrm{Cu}$ loading $\left(\mathrm{Cu}_{50}\right)$, almost total coverage of the ZnO surface is observed, both within and outside the hexagonal region, with the surface being essentially saturated with $\mathrm{Cu}$ at this point, in common with the O-terminated surfaces already discussed; the main difference is the higher proportion of the $\mathrm{ZnO}$ surface covered by $\mathrm{Cu}$ for the $\mathrm{Zn}$-terminated surface, which can be attributed simply to the fact that the Zn-terminated surface necessarily contains more $\mathrm{Zn}$ atoms which are evenly spread across the surface, rather than $\mathrm{Cu}$ atoms being initially concentrated at either $\mathrm{Zn}$ adatom or $\mathrm{O}$ vacancy sites, as was observed for the two O-terminated surfaces.

The IP-calculated adsorption energies for $\mathrm{Cu}$ clusters on this surface are also presented in Table 5. For the O-rich $\mathrm{Zn}$ terminated surface, $\mathrm{Cu}$ clusters consisting of 4 or more $\mathrm{Cu}$ atoms are adsorbed exothermically. For the smaller clusters (up to $\mathrm{Cu}_{8}$ ), there is a maximum in exothermicity of adsorption for $\mathrm{Cu}_{5}$, as larger clusters are less easily accommodated within the favoured hexagonal surface region defined by the $\mathrm{O}$ adatoms, without incurring repulsive interactions between $\mathrm{Cu}$ and surface $\mathrm{O}$. For high numbers of adsorbed $\mathrm{Cu}$ atoms (i.e. the $\mathrm{Cu}_{24}$ and $\mathrm{Cu}_{50}$ systems), the adsorption energy with respect to bulk $\mathrm{Cu}$ is even more exothermic, probably due to the extensive attractive $\mathrm{Cu}-\mathrm{Cu}$ interactions which make these systems more stable with respect to bulk $\mathrm{Cu}$. We also find that the adsorption energies with respect to bulk $\mathrm{Cu}$ are less exothermic compared to those for the O-poor O-terminated surface, which can be rationalised by considering the stronger $\mathrm{Cu}-\mathrm{Zn}$ attractive interaction for the highly undercoordinated $\mathrm{Zn}$ atoms present in the surface pits resulting from $O$ vacancies.

Zn-poor Zn-terminated surface. The fourth reconstructed $\mathrm{ZnO}$ surface is the $\mathrm{Zn}$-terminated surface featuring $\mathrm{Zn}$ vacancies (hence we will refer to this as the "Zn-poor" Zn-terminated surface), leaving pits in the surface layer containing undercoordinated $\mathrm{O}$ atoms; the remainder of the surface substantially resembles the unreconstructed Zn-terminated surface, consisting of interconnected $\mathrm{small} \mathrm{Zn}_{3}$ triangular surface platforms, intermediate sized $\mathrm{Zn}_{4}$ platforms, and a larger hexagonal $\mathrm{Zn}_{7}$ surface platform (Fig. 4). The global optimisation studies show that these flat Zn-terminated surface platforms are the favoured adsorption sites for small Cu clusters, as summarised in Tables 7 and 8; graphics for the five lowest energy structures for each $\mathrm{Cu}$ cluster size can be found in the ESI (Table S4 $\dagger$ ). For a single $\mathrm{Cu}$ atom, all of the top ranked local minimum structures feature $\mathrm{Cu}$ adsorbed in a 3-coordinate hollow site defined 


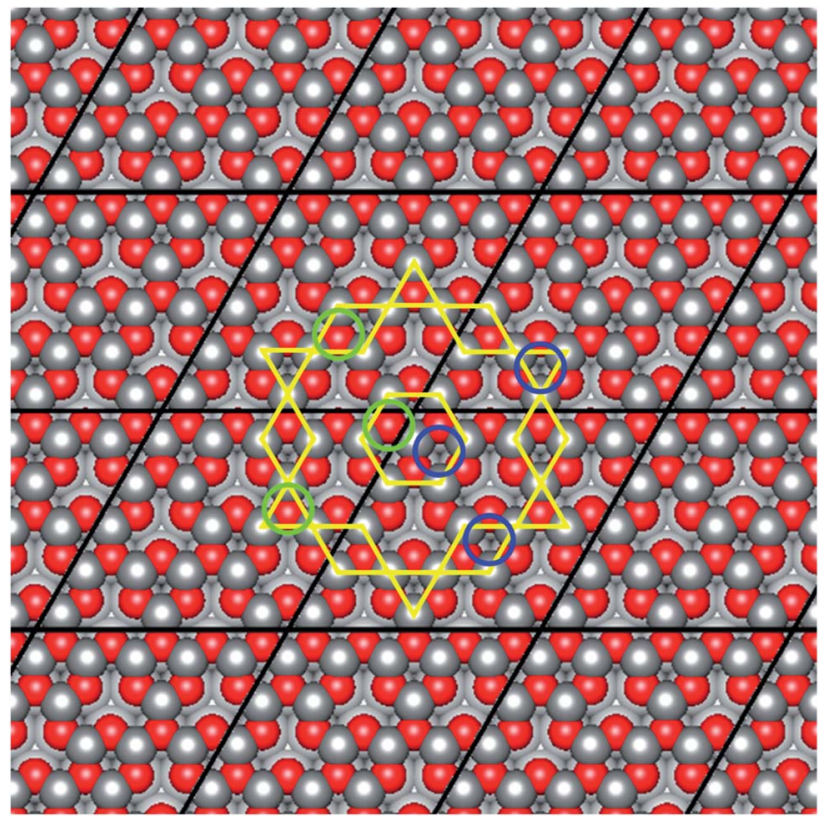

Fig. 4 Graphic illustrating the different surface adsorption sites on the reconstructed $\mathrm{Zn}$-poor $\mathrm{Zn}$-terminated surface (i.e. featuring $\mathrm{Zn}$ vacancies). The yellow lines define the three different surface platforms consisting of either 3, 4 or 7 surface $Z n$ atoms. Within each of these surface platforms, two types of 3 -coordinate $\mathrm{Zn}$ adsorption site exist, with the adsorbed $\mathrm{Cu}$ atom either eclipsing a surface $\mathrm{O}$ atom (indicated by green circles) or not (indicated by blue circles).

by surface $\mathrm{Zn}$. Two types of 3-coordinate $\mathrm{Zn}$ sites are present on the surface, either with or without the added $\mathrm{Cu}$ eclipsing a $\mathrm{O}$ atom below; the sites without $\mathrm{Cu}$ eclipsing $\mathrm{O}$ were found to be more stable, attributable to a minimisation of repulsive $\mathrm{Cu}-\mathrm{O}$ interactions. The three most stable minima consisted of structures in which the $\mathrm{Cu}$ atom is accommodated in such a site in each of the three different surface platforms identified, with the global minimum energy structure featuring the $\mathrm{Cu}$ atoms adsorbed on a triangular site. This finding can be attributed to the fact that the triangular site is bound by three $\mathrm{Zn}$ surface vacancy pits, resulting in a stronger interaction between the undercoordinated $\mathrm{O}$ atoms defining the edge of the surface pits, and the three $\mathrm{Zn}$ atoms forming the 3-coordinate adsorption site, which in turn induces a stronger interaction between the $\mathrm{Zn}$ and $\mathrm{Cu}$. Similarly, the $2^{\text {nd }}$ and $3^{\text {rd }}$ ranked structures consist of $\mathrm{Cu}$ adsorbed in the non-eclipsed site of the parallelogrammical and hexagonal surface platforms, respectively.

For two $\mathrm{Cu}$ atoms, the triangular site is too small to accommodate both $\mathrm{Cu}$ atoms comfortably, hence this site only appears as the $5^{\text {th }}$ ranked local minimum; on the other hand, the intermediate parallelogrammical surface platform is the $\mathrm{Cu}_{2}$ adsorption site for both the global minimum and $4^{\text {th }}$ ranked structure, whilst two distinct $\mathrm{Cu}_{2}$ structures are observed on the larger hexagonal platform in the $2^{\text {nd }}$ and $3^{\text {rd }}$ ranked structures. As we move to larger $\mathrm{Cu}_{3}$ and $\mathrm{Cu}_{4}$ clusters, the triangular adsorption site no longer appears in any of the top 5 ranked local minimum structures, with the hexagonal site becoming predominant for larger clusters, owing to its larger size, although for both $\mathrm{Cu}_{3}$ and $\mathrm{Cu}_{4}$, structures featuring $\mathrm{Cu}$ adsorbed on the parallelogrammical sites persist amongst the local minima. Notably, there is a conspicuous absence of 3D clusters for $\mathrm{Cu}_{4}$, in contrast with both the O-terminated reconstructed surfaces, and also the O-rich Zn-terminated surface; although for the latter, the global minimum structure did indeed feature a planar arrangement of adsorbed $\mathrm{Cu}$. For $\mathrm{Cu}_{5}$ clusters on the present reconstructed surface, 3D clusters begin to emerge, appearing as the $2^{\text {nd }}$ and $5^{\text {th }}$ ranked local minimum structures, but planar $\mathrm{Cu}$ configurations dominate. The results suggest that whilst planar $\mathrm{Cu}$ cluster formation is favourable due to the maximised interaction with the flat $\mathrm{Zn}$-terminated surface platforms (similar to that observed for the $\mathrm{Zn}$ terminated surface decorated with O-adatoms), once the $\mathrm{Zn}$ terminated surface platforms are sufficiently covered by monolayer $\mathrm{Cu}, 3 \mathrm{D} \mathrm{Cu}$ cluster formation begins to appear; that planar cluster formation is favoured until the boundaries of the Zn-terminated surface platforms are reached is reflected further by the fact that of the top 5 ranked local minimum structures for $\mathrm{Cu}_{5}$ clusters, all are located atop the large hexagonal platform, with the smaller triangular and parallelogrammical $\mathrm{Zn}$ terminated platforms being too small to accommodate $\mathrm{Cu}$ clusters of this size. The tendency towards planar $\mathrm{Cu}$ cluster formation persists for $\mathrm{Cu}_{6}$, although the relative energies presented in Table 8 show that 3D Cu cluster formation is more competitive compared to that for $\mathrm{Cu}_{5}$; for both $\mathrm{Cu}_{5}$ and $\mathrm{Cu}_{6}$, the global minimum structure features a planar $\mathrm{Cu}$ cluster, whilst the second ranked local minimum structure shows a $3 \mathrm{D} \mathrm{Cu}$ cluster geometry; however for $\mathrm{Cu}_{6}$, the $1^{\text {st }}$ and $2^{\text {nd }}$ ranked structures are closer in energy by $\sim 0.15 \mathrm{eV}$ compared to that for $\mathrm{Cu}_{5}$. This trend continues for $\mathrm{Cu}_{7}$ and $\mathrm{Cu}_{8}$, with the planar cluster geometry remaining evident in the global minimum, but for increasing amounts of $\mathrm{Cu}$, the alternative $3 \mathrm{D} \mathrm{Cu}$ cluster geometry becomes increasingly competitive, with the $1^{\text {st }}$ and $2^{\text {nd }}$ ranked structures for $\mathrm{Cu}_{8}$ being separated by only $0.05 \mathrm{eV}$.

For larger $\mathrm{Cu}_{24}$ clusters, the emergence of $3 \mathrm{D} \mathrm{Cu}$ clusters is clearly evident, as expected for such a high $\mathrm{Cu}$ loading. The global minimum structure and the $2^{\text {nd }}$ ranked local minimum structure both show discreet $\mathrm{Cu}$ clusters, whilst the remaining structures show coalescence of the Cu clusters, forming bars of $\mathrm{Cu}$ across the periodically repeated surface; however we see that the discreet $\mathrm{Cu}_{24}$ clusters are more stable, with the $2^{\text {nd }}$ ranked structure being within only $0.03 \mathrm{eV}$ of the global minimum, whilst the $3^{\text {rd }}$ ranked structure, which exhibits coalescence of the $\mathrm{Cu}$ clusters is $0.22 \mathrm{eV}$ less stable than the global minimum. In all of the top ranked structures, we find that the $\mathrm{Cu}$ clusters form as a result of the cluster spreading beyond the confines of the distinct flat $\mathrm{ZnO}$ surface platforms identified, with clusters on the large hexagonal and intermediate parallelogrammical platforms merging together. Whilst the large size of the $\mathrm{Cu}_{24}$ cluster means that some $\mathrm{O}$ vacancy surface pits are covered by the $\mathrm{Cu}$ cluster, we still find that the boundaries of the flat surface platforms largely define the boundaries of the $\mathrm{Cu}$ cluster. For the very high $\mathrm{Cu}$ loading in the $\mathrm{Cu}_{50}$ system, the surface is essentially saturated with $\mathrm{Cu}$, in common with the other three reconstructed surfaces investigated. For each of the top 5 ranked structures, the regions of the surface not covered 
Table 7 Graphics for lowest energy structures obtained for adsorbed Cu clusters of various sizes. See ESI for the remaining 4 candidate local minimum structures (Table S4). Blue spheres represent $\mathrm{Cu}$, whilst red and grey spheres represent $\mathrm{O}$ and $\mathrm{Zn}$, respectively. The top ZnO layer (i.e. involved in the reconstruction) atoms are highlighted with darker spheres, whilst the subsurface $\mathrm{ZnO}$ atoms are represented by faded spheres. Thin green lines indicate cell boundaries. IP-calculated adsorption energies with respect to bulk Cu are listed below the graphics for each lowest energy structure

1

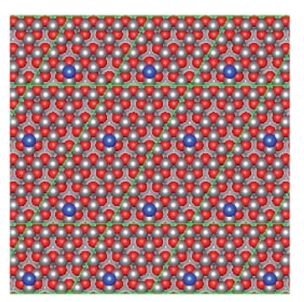

1.873

6

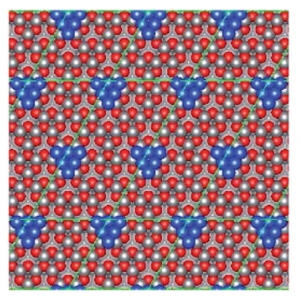

0.187
2

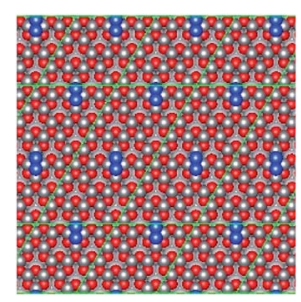

0.760

7

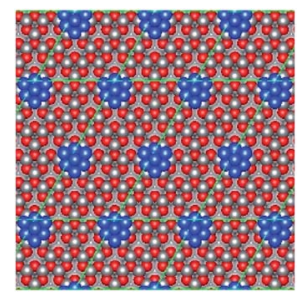

No. $\mathrm{Cu}$

3

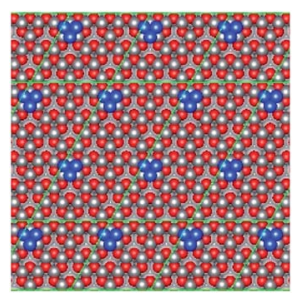

Adsorption energy w.r.t. bulk $\mathrm{Cu} / \mathrm{eV}$

0.424

No. Cu

8

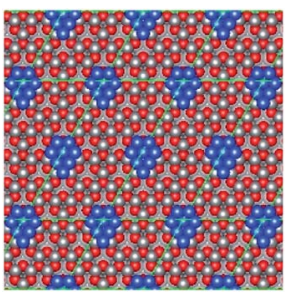

4

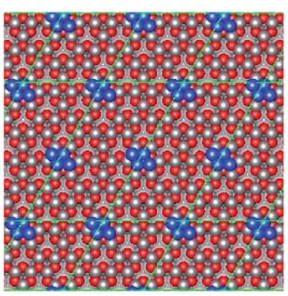

0.303

24

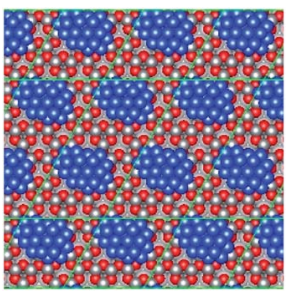

5

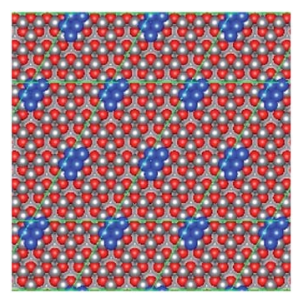

0.227

Adsorption energy w.r.t. bulk $\mathrm{Cu} / \mathrm{eV}$

0.160

0.185

0.136

0.052

by $\mathrm{Cu}$ correspond to the Zn-vacancy sites; however it is notable that the global minimum structure shows a greater degree of $\mathrm{Cu}$ wetting, with only two $\mathrm{Zn}$ surface vacancies being left exposed, compared to the remaining 4 local minima in which three surface $\mathrm{Zn}$ surface vacancies remain visible and are not subducted beneath the $\mathrm{Cu}$ layer. This observation suggests that for high $\mathrm{Cu}$ surface concentrations, $\mathrm{Cu}-\mathrm{Cu}$ interactions become relatively more important in determining cluster morphology, with any repulsive $\mathrm{Cu}-\mathrm{O}$ interactions arising from coverage of the $\mathrm{Zn}$ surface vacancies being compensated by the favourable $\mathrm{Cu}-\mathrm{Cu}$ interactions.

The IP adsorption energies for the Cu clusters on this surface with respect to bulk $\mathrm{Cu}$ are also presented in Table 7 . The general trend in adsorption energies resembles that of the Zn-rich O-terminated surface, with adsorption being endothermic across the full range of $\mathrm{Cu}$ cluster sizes, but becoming less so for larger $\mathrm{Cu}$ clusters, which can be attributed to the greater number of attractive $\mathrm{Cu}-\mathrm{Cu}$ interactions for larger $\mathrm{Cu}$ clusters. The exception to this trend is the $\mathrm{Cu}_{7}$ cluster, which is the least endothermically adsorbed of all of the smaller $\mathrm{Cu}$ clusters up to $\mathrm{Cu}_{8}$, which is adsorbed slightly more endothermically, which can simply be attributed to the limited size of the hexagonal $\mathrm{Zn}$-terminated surface platform that serves as the favoured adsorption site; the larger $\mathrm{Cu}_{8}$ cluster spills over the boundaries of this site, thus incurring a repulsive $\mathrm{Cu}-\mathrm{O}$ interaction as the additional $\mathrm{Cu}$ is forced closer to the undercoordinated $\mathrm{O}$ associated with the $\mathrm{Zn}$ vacancy.

Table 8 Relative energies (in eV) for the 5 lowest energy structures for adsorbed Cu clusters of various sizes. The tabulated values are calculated with respect to the global minimum energy structure. See ESI for corresponding structures (Table S4)

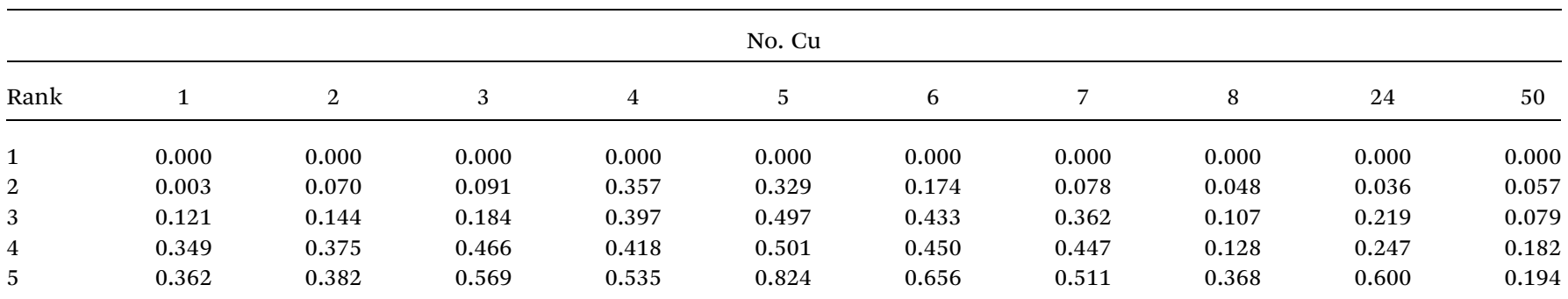




\section{DFT refinement of IP structures}

Having obtained structures for the five lowest energy IP structures for each of the surface reconstructions and a variety of $\mathrm{Cu}$ cluster sizes from the global optimisation studies, DFT refinement of these structures is required to verify the validity of the global optimisation approach, and to allow for a more detailed analysis. For this purpose, the $\mathrm{Cu}_{8}$ clusters for each of the four surface reconstructions were subject to DFT refinement. In alignment with previous studies, ${ }^{15,21}$ it was found that, in general, the morphology of the IP optimised structures is retained by the DFT optimisation and that the lowest minimum IP structure is also found to be the most stable local minimum after DFT refinement, therefore confirming that the potentials used in this study for the exploration of the potential energy surface are suitable for these types of systems. A full discussion is provided in the ESI. $\uparrow$ Nonetheless, there are a few important instances where the DFT refined systems deviate from the IP structures and relative stability ordering of lowest energy minima, which we will discuss here.

For both of the O-terminated surfaces, and for the Zn-poor Zn-terminated surface, good agreement between the IP and DFT optimised structures and ordering of relative stability of low energy structures is obtained. However, for the O-rich $\mathrm{Zn}$ terminated surface, DFT refinement results in a significant deviation in terms of both $\mathrm{Cu}$ cluster structure, and relative stability (Table 9). Perhaps the two most striking structural features evident post-refinement are the close proximity of $\mathrm{Cu}$ and surface $\mathrm{O}$ adatoms, and the considerable displacement of these surface $\mathrm{O}$ adatoms with respect to their positions in the IP optimised structures; during IP optimisation, these atoms largely remain unperturbed from their positions on the clean IP optimised surfaces. These differences can be rationalised by considering that the highly undercoordinated surface $\mathrm{O}$ adatoms are likely to be considerably less reduced compared to their bulk and subsurface counterparts, owing to having fewer $\mathrm{Zn}$ neighbours. Not only would this factor reduce the repulsive interaction between $\mathrm{Cu}$ and $\mathrm{O}$ implied by the potentials used (i.e. both species being comparatively electron rich), but also open the possibility for electron transfer from $\mathrm{Cu}$ to $\mathrm{O}$ (that is to say, partial $\mathrm{Cu}$ oxidation), which clearly cannot be accounted for explicitly using the IP unlike the DFT approach. In tandem, these two conclusions support the observed changes upon DFT refinement, which suggest a stronger and more attractive interaction between the surface $\mathrm{O}$ adatoms and the $\mathrm{Cu}$ cluster atoms.

To examine the extent of charge transfer between $\mathrm{Cu}$ and surface $\mathrm{O}$ adatoms, Bader charge analysis was performed for the lowest energy DFT-optimised structure (corresponding to the $5^{\text {th }}$ ranked IP structure). The calculated Bader charges show a moderate decrease in electronic charge compared to bulk $\mathrm{Cu}$ for all except two of the $\mathrm{Cu}$ atoms, with $\Delta \beta$ ranging from $-0.17 e$ to $-0.28 e$, averaging $-0.23 e$. The remaining two $\mathrm{Cu}$ atoms showed negligible changes in the Bader charges with respect to bulk $\mathrm{Cu}$, with $\Delta \beta=-0.00017$ and $\Delta \beta=+0.003$. As illustrated in Fig. 5 , the two $\mathrm{Cu}$ atoms with least significant $\Delta \beta$ are the only two $\mathrm{Cu}$ atoms coordinated only by other $\mathrm{Cu}$ atoms, and not coordinated with any surface $\mathrm{O}$ adatoms, strongly suggesting

Table 9 Structure and relative stability for the top 5 ranked low energy IP structures for $\mathrm{Cu}_{8}$ clusters adsorbed on the reconstructed $\mathrm{O}$-rich $\mathrm{Zn}$ terminated $\mathrm{ZnO}$ surface, before and after DFT refinement

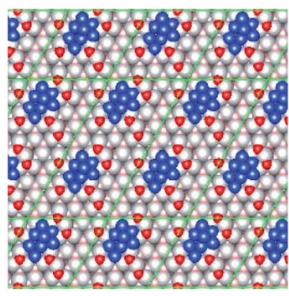

0.000

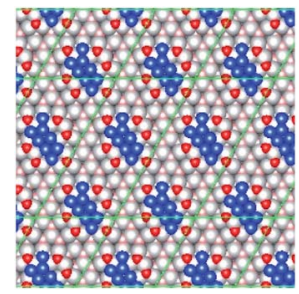

0.054
IP rank

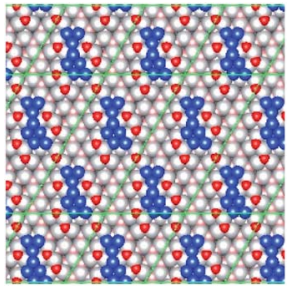

IP relative energy/eV

0.113

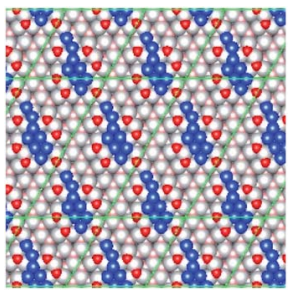

0.132
5

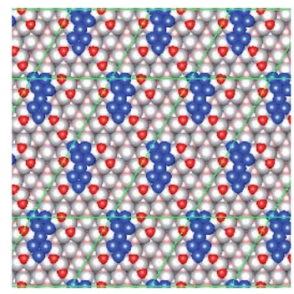

0.201
4

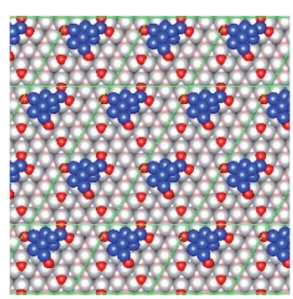

2

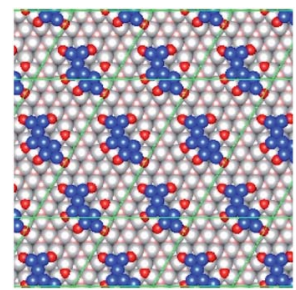

DFT rank

5

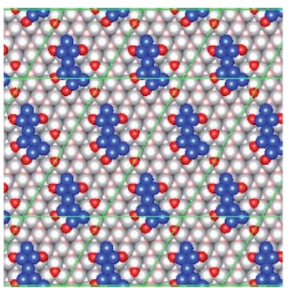

DFT relative energy/eV
3

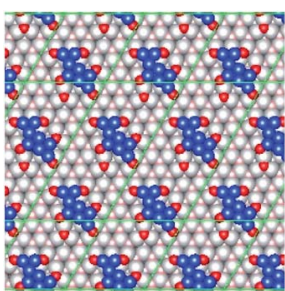

1

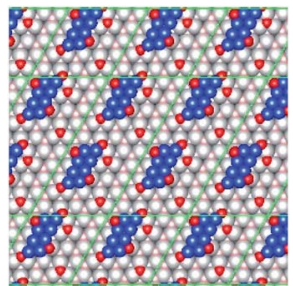

1.183

0.000 


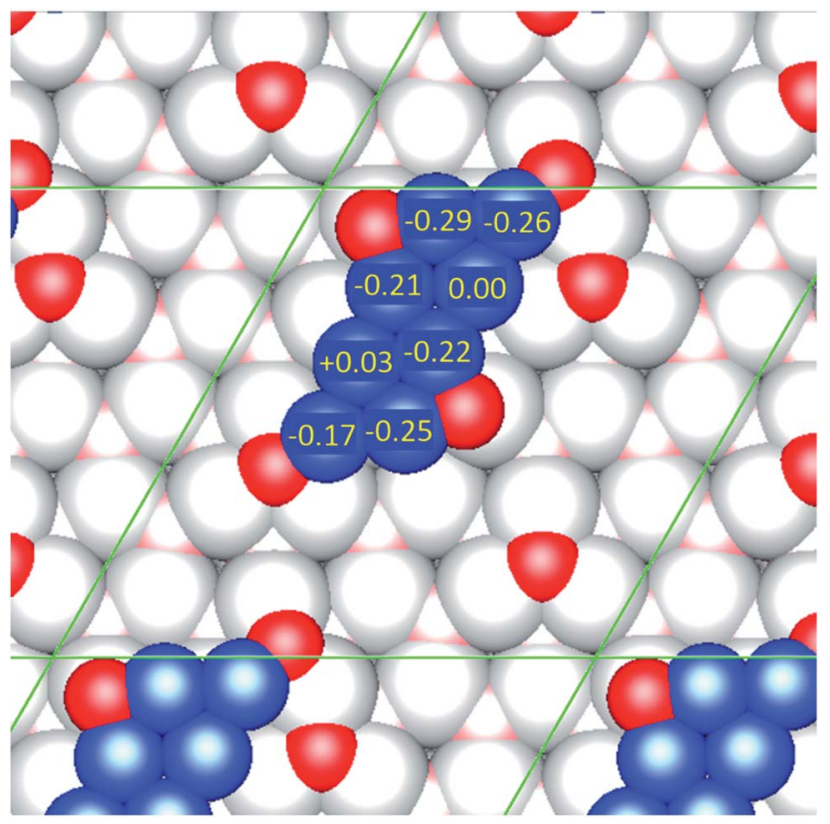

Fig. 5 Graphic depicting the lowest energy DFT-optimised structure for $\mathrm{C} \mathrm{Cu}_{8}$ cluster adsorbed on the O-rich $\mathrm{Zn}$-terminated reconstructed polar $\mathrm{ZnO}$ surface. The values superimposed on the $\mathrm{Cu}$ atoms correspond to $\Delta \beta$, the difference in the calculated Bader charge for that $\mathrm{Cu}$ atom relative to bulk $\mathrm{Cu}$.

that charge transfer from coordinating $\mathrm{Cu}$ atoms via surface $\mathrm{O}$ adatoms plays a key role in stabilisation of the adsorbed $\mathrm{Cu}$ cluster.

The higher mobility of the surface $\mathrm{O}$ adatoms (which may be attributed both to a weaker $\mathrm{O}-\mathrm{Zn}$ interaction and stronger $\mathrm{Cu}-\mathrm{O}$ interaction for these surface $\mathrm{O}$ adatoms compared to that assumed by the IP approach) appears to play an important role in the re-ordering of the lowest energy structures after DFT optimisation. For the IP optimised $\mathrm{Cu}_{8}$ clusters, the surface $\mathrm{O}$ adatoms present a barrier to the favoured planar $\mathrm{Cu}$ cluster growth across the flat Zn-terminated surface observed for smaller $\mathrm{Cu}$ clusters, hence the IP global minimum features a 3D $\mathrm{Cu}$ cluster (consisting of a highly coordinated $\mathrm{Cu}_{7}$ planar base, with an additional $\mathrm{Cu}$ atom on top), whilst the remaining 4 lowest energy structures all feature flat $\mathrm{Cu}_{8}$ clusters that compromise $\mathrm{Cu}-\mathrm{Cu}$ coordination in order to minimise close contact with the $\mathrm{O}$ surface adatoms. However, upon DFT refinement, no such condition appears to be present, hence the DFT global minimum (which was obtained from refinement of the $5^{\text {th }}$ lowest energy IP structure) features a distinctive planar $\mathrm{Cu}$ cluster with a minimal perimeter (maximising $\mathrm{Cu}-\mathrm{Cu}$ coordination), with the surface $\mathrm{O}$ adatoms positions at the corners of the flat $\mathrm{Cu}$ cluster (i.e. interacting with the least coordinated $\mathrm{Cu}$ atoms). By contrast, the IP global minimum structure is only the $4^{\text {th }}$ lowest energy DFT structure (whilst retaining its 3D morphology), with only the $3^{\text {rd }}$ lowest energy IP structure being found to be less stable upon DFT refinement, which can be attributed to the low $\mathrm{Cu}-\mathrm{Cu}$ coordination evident in the initial IP structure, which is preserved upon DFT optimisation.
Clearly, in this case, the IP global optimisation approach cannot be used alone to approximate DFT global minimum energy structures. However, the present results illustrate the limitations of the approach and provide key insights that will be invaluable in the tailoring of the interatomic potentials to meet the needs of more complex systems.

Another interesting observation pertains to the O-poor $\mathrm{Zn}$ terminated surface. Whilst the overall trend in stability is reproduced, with the lowest energy IP structure being found to also be the lowest energy DFT structure, the DFT-refined lowest minimum structure is considerably more stable than the other low-energy DFT-refined structures compared to the IP relative energies. The enhanced stability of the DFT global minimum structure compared to its IP counterpart can be linked to the lesser degree of $\mathrm{O}$ reduction for the $\mathrm{O}$ atoms that define the $\mathrm{Zn}$ surface vacancy pit. Whilst the highly coordinated planar $\mathrm{Cu}_{8}$ structure is largely retained after DFT optimisation of the IP global minimum, we see that the position of the cluster has shifted such that the least coordinated $\mathrm{Cu}$ atom (having only 2 $\mathrm{Cu}$ neighbours) is located within the $\mathrm{Zn}$ vacancy pit, implying a degree of $\mathrm{Cu}$ oxidation from interaction with the $\mathrm{O}$ atoms within the surface pit that lack a full complement of $\mathrm{Zn}$ neighbours (Fig. 6). To test this, Bader charge analysis was performed; we find that the $\mathrm{Cu}$ atom located within the $\mathrm{Zn}$ vacancy surface pit is significantly more oxidised than the remaining $7 \mathrm{Cu}$ atoms in the cluster, with a Bader charge increase of $\Delta \beta=-0.57 e$, with respect to a single isolated neutral $\mathrm{Cu}$ atom. By comparison, for the remaining $7 \mathrm{Cu}$ atoms, $\Delta \beta$ ranges from $-0.21 e$ to $+0.06 e$, averaging $\Delta \beta=-0.09 e$, consistent with essentially unoxidized $\mathrm{Cu}^{0}$, with this small deviation being attributable to the charge delocalisation owing to the metallic character of $\mathrm{Cu}$. The $\Delta \beta$ calculated for the $\mathrm{Cu}$ atom located within the $\mathrm{Zn}$ vacancy site is consistent with previous studies which determined the Bader charges for $\mathrm{Cu}$ and $\mathrm{O}$ in bulk $\mathrm{Cu}_{2} \mathrm{O}$, determining $\Delta \beta=-0.53 e$ using the PBE GGA functional. ${ }^{44}$ Hence, there is strong evidence that in the present system, the $\mathrm{Cu}$ atom which is located within the $\mathrm{Zn}$ surface vacancy site after DFT refinement corresponds to a $\mathrm{Cu}^{+}$cation, in line with previous DFT studies which suggest that oxidising $\mathrm{Cu}$ atoms within surface $\mathrm{Zn}$ vacancy sites can serve as anchor sites for Cu cluster growth. ${ }^{26}$ This finding is consistent with our rationalisation of the more drastic changes observed for DFT refinement of the $\mathrm{Cu}$ clusters on top of the O-rich $\mathrm{Zn}$-terminated surface. For the other DFT refined structures, no such migration of the $\mathrm{Cu}$ cluster to accommodate one $\mathrm{Cu}$ atom in a $\mathrm{Zn}$ vacancy site is observed, which is reflected in the gap in stability between the global minimum and all 4 of the remaining DFT optimised structures. Nonetheless, in all of the other structures, it is still the case that after DFT refinement, the $\mathrm{Cu}$ clusters adopt slightly more planar clusters; whilst none of the cluster geometries deviate significantly from their IP optimised positions atop the large hexagonal surface ZnO platform, the DFT optimised structures show $\mathrm{Cu}$ atoms much closer to the edges of the hexagonal platform, decreasing the distance between the $\mathrm{Cu}$ atoms at the edge of the cluster and the undercoordinated $\mathrm{O}$ atoms defining the edges of the $\mathrm{Zn}$ vacancy pits. This result further suggests that the interaction between $\mathrm{Cu}$ and 

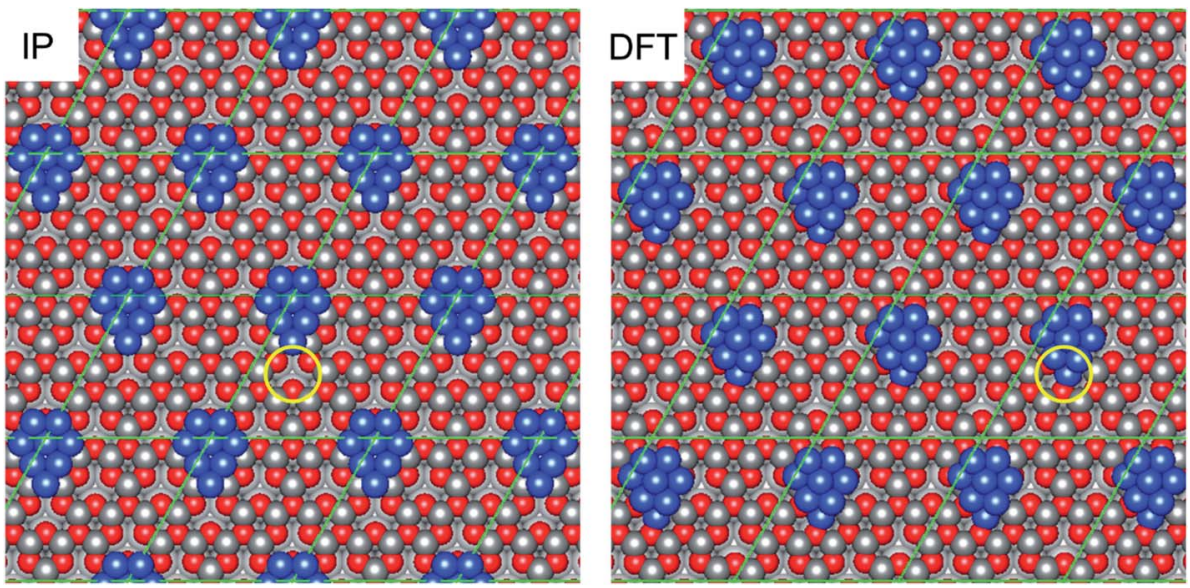

Fig. 6 Graphics depicting the lowest energy IP structure (left) and the DFT-refined lowest energy structure (right) for a Cu $\mathrm{Cluster}$ adsorbed on a $\mathrm{Zn}$-poor $\mathrm{Zn}$-terminated surface (i.e. featuring $\mathrm{Zn}$ vacancies). Note the shift of the $\mathrm{Cu}_{8}$ cluster such that one of the $\mathrm{Cu}$ atoms is located within the $\mathrm{Zn}$ vacancy in the DFT-refined structure (indicated with yellow circles). Thin green lines indicate cell boundaries.

undercoordinated surface $\mathrm{O}$ is perhaps not as repulsive as that described using IP methods, agreeing with the rationalisation for the impact of DFT refinement on the O-rich Zn-terminated surface.

For comparison, Bader charge analysis was also performed on the lowest energy DFT optimised $\mathrm{Cu}_{8}$ configuration for the remaining two surfaces. Whilst we do not observe the same extent of $\mathrm{Cu}$ oxidation observed for the $\mathrm{Cu}$ atom located within the $\mathrm{Zn}$ vacancy of the $\mathrm{Zn}$-poor $\mathrm{Zn}$-terminated surface, a similar trend is noted whereby more significant charge transfer from $\mathrm{Cu}$ atoms is observed for $\mathrm{Cu}$ atoms which are more highly coordinated with surface $\mathrm{O}$ atoms.

For the O-poor O-terminated surface, the most stable DFT structure consists of the $\mathrm{Cu}_{8}$ located largely within the surface pits arising from the $\mathrm{O}$ vacancies, with most of the $\mathrm{Cu}$ atoms lying below the topmost $\mathrm{ZnO}$ layer. Bader charge analysis reveals that whilst the topmost three $\mathrm{Cu}$ atoms are not significantly oxidised (average $\Delta \beta=-0.04 e$ ), the $5 \mathrm{Cu}$ atoms located mostly below the topmost $\mathrm{ZnO}$ layer were significantly more so (average $\Delta \beta=-0.28 e$ ). For the topmost $\mathrm{ZnO}$ layer, whilst the Bader analysis showed little deviation between the electron count for surface $\mathrm{O}$ atoms and that of $\mathrm{O}$ atoms in the $\mathrm{ZnO}$ bulk, some of the surface $\mathrm{Zn}$ atoms were considerably reduced compared to $\mathrm{Zn}$ atoms in bulk $\mathrm{ZnO}$ (average $\Delta \beta=+0.31 e$ for the 3 most reduced $\mathrm{Zn}$ atoms). This suggests that stabilisation of the $\mathrm{Cu}$ cluster involves some degree of charge transfer from $\mathrm{Cu}$ to surface $\mathrm{Zn}$ atoms which are necessarily undercoordinated owing to the missing surface $\mathrm{O}$ atoms arising from the vacancy. Whilst the electron count values are insufficient to suggest full oxidation of $\mathrm{Cu}$ by surface $\mathrm{Zn}$, the observations are consistent with delocalisation of electron density between the adsorbed $\mathrm{Cu}$ and the less oxidised undercoordinated $\mathrm{Zn}$ atoms which are thus more metal-like.

For the Zn-rich O-terminated surface, we see a very similar trend, with three of the eight adsorbed $\mathrm{Cu}$ atoms having small $\Delta \beta$ (average $+0.02 e$ ), and the remaining five being moderately oxidised (average $\Delta \beta=-0.27 e$ ). Again, the three relatively unperturbed $\mathrm{Cu}$ atoms are located in the topmost layer of the 3D Cu cluster observed on this surface, and thus have no direct contact with the surface $\mathrm{ZnO}$ atoms, thus more closely resembling bulk $\mathrm{Cu}$. Conversely, for the surface $\mathrm{Zn}$ adatoms, whilst four of the six present have relatively small $\Delta \beta$ values relative to $\mathrm{Zn}$ atoms in bulk $\mathrm{ZnO}$ (average $\Delta \beta=+0.08$ ), the remaining two surface $\mathrm{Zn}$ atoms show a marked increase in electronic charge (average $\Delta \beta=+0.60 e$ ). These two surface $\mathrm{Zn}$ adatoms are those directly adjacent to the adsorbed $3 \mathrm{D} \mathrm{Cu}$ cluster, strongly suggesting that undercoordinated surface $\mathrm{Zn}$ adatoms are able to stabilise the $\mathrm{Cu}$ cluster by accepting electrons from $\mathrm{Cu}$.

Overall, the Bader charge analyses show that there is indeed an electronic component to the stabilisation of adsorbed $\mathrm{Cu}$ clusters, and that the surface features arising from reconstruction of the polar ZnO surfaces, namely $\mathrm{O}$ and $\mathrm{Zn}$ adatoms and vacancies, are of importance in mediating this stabilisation mechanism.

\section{Discussion}

\section{Structure of $\mathrm{Cu}$ clusters adsorbed on reconstructed polar ZnO surfaces}

The global optimisation studies show very clearly that the $\mathrm{Cu}$ cluster morphology is closely linked to the different surface sites presented by different $\mathrm{ZnO}$ surface reconstructions. In general, we find that, at least for smaller $\mathrm{Cu}$ clusters, planar growth is favoured on the Zn-terminated surface, whilst 3D cluster growth is favoured for the O-terminated surfaces. This difference can be attributed to the highly attractive $\mathrm{Cu}-\mathrm{Zn}$ interaction modelled by the IP method used; in the absence of any surface reconstruction, the Zn-terminated surface presents a flat plane of surface $\mathrm{Zn}$ atoms, hence $\mathrm{Cu}$ clusters form by spreading evenly over this surface to maximise the attractive $\mathrm{Cu}-\mathrm{Zn}$ interaction, with the surface features arising from the reconstruction ( $\mathrm{Zn}$ vacancies and $\mathrm{O}$ adatoms) merely serving to impede complete $\mathrm{Cu}$ monolayer formation due to the repulsive $\mathrm{Cu}-\mathrm{O}$ interactions. On the other hand, the unreconstructed O- 
terminated surface conversely presents as a flat plane of $\mathrm{O}$ atoms which is not conducive to $\mathrm{Cu}$ cluster growth; however the surface features arising from reconstruction ( $O$ vacancies and $\mathrm{Zn}$ adatoms) provide undercoordinated $\mathrm{Zn}$ atoms at the surface which can serve as a nucleation site for 3D $\mathrm{Cu}$ cluster growth.

Whilst the observed $\mathrm{Cu}$ cluster morphology determined in the present work appears to contradict previous experimental STM studies, which reported 3D Cu cluster growth on the Znterminated surfaces, ${ }^{19}$ and $2 \mathrm{D}$ planar $\mathrm{Cu}$ cluster growth on the O-terminated surface, ${ }^{20}$ the rationalisation for the observed cluster morphologies in these works and the present work has, in fact, the same basis, and the apparent contradiction arises from the difference in cluster size. Dulub et al. rationalise the 3D $\mathrm{Cu}$ cluster growth over the reconstructed Zn-terminated surface as being the result of a high kinetic barrier to extensive planar $\mathrm{Cu}$ cluster growth resulting from repulsive interactions between $\mathrm{Cu}$ and undercoordinated surface $\mathrm{O}$ at surface $\mathrm{ZnO}$ terrace edges and $\mathrm{Zn}$ vacancy pits, implying then that $\mathrm{Cu}$ cluster growth is indeed 2D planar up until the point at which the $\mathrm{Cu}$ monolayer becomes larger enough to reach the barriers imposed by the undercoordinated surface $\mathrm{O}$ atoms, at which point subsequent $\mathrm{Cu}$ cluster growth results in the 3D clusters observed experimentally. ${ }^{19}$ This argument is entirely consistent with the observed patterns of $\mathrm{Cu}$ cluster growth on the $\mathrm{Zn}$ terminated surface obtained from the global optimisation studies performed in the present work; for both the O-adatom and Zn-vacancy reconstructions, planar $\mathrm{Cu}$ growth does indeed occur for smaller numbers of $\mathrm{Cu}$ atoms, and as can be seen for the larger $\mathrm{Cu}_{8}, \mathrm{Cu}_{2}$ and $\mathrm{Cu}_{50}$ systems, 3D $\mathrm{Cu}$ cluster growth does indeed take place instead of continued planar cluster growth once the $\mathrm{Cu}$ cluster meets the boundaries imposed by the undercoordinated surface $\mathrm{O}$ atoms. This effect is particularly noticeable for the $\mathrm{Zn}$-poor $\mathrm{Zn}$ terminated surface; $\mathrm{Cu}$ cluster growth is almost exclusively planar up to and including the $\mathrm{Cu}_{8}$ system, whilst the $\mathrm{Cu}_{24}$ cluster shows distinct $3 \mathrm{D}$ growth, and the boundaries of the $\mathrm{Cu}_{24}$ are observed to be consistent with that defined by the edges of the surface $\mathrm{Zn}$ vacancy pits which consist of undercoordinated $\mathrm{O}$ atoms. Hence, the apparent contradiction between the present work and previous experimental studies is merely a consequence of the inability of experimental studies to observe $\mathrm{Cu}$ cluster growth at a level of sequential atomic growth of small clusters, and conversely the limitation imposed by ZnO surface cell size in the present work that prohibits the modelling of very large $\mathrm{Cu}$ clusters confined to ZnO surface terraces, as observed in the experimental studies. Taken in tandem, however, the present work and the previous STM studies provide a detailed illumination of the factors controlling $\mathrm{Cu}$ cluster growth on the reconstructed $\mathrm{Zn}$-terminated polar (0001) surface. Hence, upon closer inspection, there is in fact no true contradiction between the previous STM studies and the present work, since the $\mathrm{Cu}$ cluster sizes investigated are of vastly different orders of magnitude. The rationalisation for the observed $\mathrm{Cu}$ cluster morphologies put forward in the present work confirm those suggested by the previous experimental studies, illustrating how these factors can affect the structures of small and large adsorbed $\mathrm{Cu}$ clusters in different ways.
The findings of the present work concerning $\mathrm{Cu}$ cluster morphology over the O-terminated reconstructed polar $\mathrm{ZnO}$ surface are more difficult to reconcile with those of the experimental STM studies, owing to the various technical challenges inherently associated with the O-terminated polar $\mathrm{ZnO}$ surface. ${ }^{19,20}$ Whilst the experimental studies concluded that planar $\mathrm{Cu}$ cluster growth takes place on the O-terminated surface, a maximum $\mathrm{Cu}$ cluster height of $\sim 4.7 \AA$ was observed, which is consistent with $\mathrm{Cu}$ clusters consisting of more than one layer of adsorbed $\mathrm{Cu}$, and corresponds well with the 3D Cu clusters on the O-terminated surface obtained in the present work, such as the $\mathrm{Cu}_{24}$ cluster adsorbed on the reconstructed O-terminated surface featuring $\mathrm{Zn}$ adatoms, for which the topmost $\mathrm{Cu}$ atoms were positioned at $4.17 \AA$ above the topmost complete $\mathrm{ZnO}$ surface layer and consisted of 2-3 layers of $\mathrm{Cu}$ atoms. Moreover, it was observed for this system that the strong $\mathrm{Cu}-\mathrm{Zn}$ interaction between the cluster and the surface resulted in the surface $\mathrm{Zn}$ atoms being lifted above their equilibrium positions on the clean surface, hence with respect to the clean O-terminated surface, the height of the topmost $\mathrm{Cu}$ atoms would be even greater than $4.17 \AA$, bringing the computationally determined cluster height closer to the experimentally reported value. It is possible, then, that the apparent contradiction between the $\mathrm{Cu}$ cluster height and supposed planar $\mathrm{Cu}$ cluster growth observed in the experimental STM studies could essentially be a result of saturation of the O-terminated surface with $\mathrm{Cu}$, resulting in a coalescence of discrete $\mathrm{Cu}$ clusters ( $c f$. the $\mathrm{Cu}_{50}$ systems), which might present experimentally as a flat surface consisting of 2 or 3 layers of adsorbed $\mathrm{Cu}$. In any case, whilst the comparison between the STM studies and the computational results in the present work may be less complete for the O-terminated surface than for the Zn-terminated surface, the rationalisation for the $3 \mathrm{D} \mathrm{Cu}$ cluster growth on the O-terminated surfaces in the present work is simply a consequence of the well-established attractive $\mathrm{Cu}-\mathrm{Zn}$ interaction, and the presence of surface sites featuring undercoordinated $\mathrm{Zn}$ (i.e. $\mathrm{Zn}$ adatoms or $\mathrm{O}$ vacancies) which were found to be the favoured sites for $\mathrm{Cu}$ cluster growth, as opposed to $\mathrm{Cu}$ atoms spreading over the surface to maximise $\mathrm{Cu}-\mathrm{Zn}$ interactions as observed for the Zn-terminated surfaces, thus promoting 3D Cu cluster growth. Once again, it can be seen that the conclusions drawn from our studies are consistent with those obtained from previous experimental studies; hence, no true contradiction between the present work and these previous studies exist, with any apparent discrepancies linked to the vastly different $\mathrm{Cu}$ cluster sizes being modelled here, compared with those being observed in STM studies.

\section{Adsorption of $\mathrm{Cu}$ clusters}

The IP calculated adsorption energies for the global minimum structures with respect to bulk $\mathrm{Cu}$ show that $\mathrm{Cu}$ clusters of the same size are more strongly adsorbed on the O-poor Oterminated surface, and on the O-rich Zn-terminated surface, compared to the remaining other two reconstructed surfaces. In both cases, this behaviour can be attributed to these reconstructions affording undercoordinated $\mathrm{Zn}$ environments that 
maximise the opportunity for attractive $\mathrm{Cu}-\mathrm{Zn}$ interactions upon adsorption of the $\mathrm{Cu}$ cluster. However, whilst the $\mathrm{Cu}$ cluster adsorption energies with respect to bulk $\mathrm{Cu}$ were found to be endothermic for the Zn-rich O-terminated and Zn-poor Znterminated surfaces, this finding can be attributed to the high energetic cost of cleaving small $\mathrm{Cu}$ clusters from bulk, hence we might expect small, isolated $\mathrm{Cu}$ nanoclusters to interact strongly and exothermically with the reconstructed polar $\mathrm{ZnO}$ surfaces discussed in the present work. As we might expect that, under real conditions, reconstructed surfaces will feature a mixture of surface sites resembling the various $\mathrm{Zn}$ and $\mathrm{O}$ adatom and vacancy sites explored in the present work, we can expect that $\mathrm{Cu}$ cluster growth might take place at any of these sites.

\section{Validation of IP approach}

The interatomic potentials used in the present work have already been demonstrated to be highly successful in obtaining low-energy structures for $\mathrm{Cu}$ clusters adsorbed on the non-polar ZnO surface, and are an effective means of screening potential structures in a computationally expedient manner. However, the reconstructed polar $\mathrm{ZnO}$ surface explored in the present work shows surface features that consist of undercoordinated $\mathrm{Zn}$ and $\mathrm{O}$ atoms that deviate considerably from typical surface and bulk $\mathrm{Zn}$ and $\mathrm{O}$ coordination environments present in the non-polar ZnO surface that was used as a model for fitting the $\mathrm{Cu}-\mathrm{ZnO}$ potentials. Hence, it is of interest to assess the extent to which these potentials successfully identify global and local minima which are reproduced upon DFT refinement. Overall, the results show that in most cases, these potentials perform very well, with the global minimum energy IP structure for $\mathrm{a} \mathrm{Cu}_{8}$ cluster being retained upon DFT optimisation for three of the four surface reconstructions investigated. Furthermore, in those cases, the general trend in stability for the remaining top 4 local minima was broadly reproduced, with only minor changes in stability ordering being observed. The O-rich $\mathrm{Zn}$ terminated surface was the only surface reconstruction for which the IP approach failed to identify a global minimum energy structure or retain any reasonable agreement in terms of stability ordering of the local minimum energy structures upon DFT refinement. This problem can be attributed to the highly exothermic electron affinity of $\mathrm{O}$, resulting in stronger $\mathrm{Cu}-\mathrm{O}$ attractive interactions than that supposed by the IP approach, owing to partial oxidation of $\mathrm{Cu}$ by undercoordinated surface $\mathrm{O}$ adatoms, which is also reflected in the significant displacement of the surface $\mathrm{O}$ adatoms from their positions on the clean surface, since any energetic cost associated with this process is compensated by exothermic electron transfer from $\mathrm{Cu}$ to $\mathrm{O}$ adatoms. Furthermore, it is noted that the IP approach utilised $\mathrm{Cu} / \mathrm{ZnO}$ potentials fitted to DFT calculations performed for small $\mathrm{Cu}$ clusters approaching relaxed, unreconstructed, nonpolar ZnO (1010) and (1120) surfaces, and thus were not fitted to systems that have significantly undercoordinated surface $O$ atoms, which are present in some of the reconstructed surfaces discussed in the present work. Hence, given that potentials used do not account for undercoordinated surface $\mathrm{O}$ atoms, which can be understood to be less reduced than typical surface $O$ atoms that have more $\mathrm{Zn}$ neighbours, it is worth considering that perhaps an IP approach including potentials for $\mathrm{Cu}^{+}$and $\mathrm{Cu}^{2+}$ would perform far better in identifying global minima which are reproduced upon DFT refinement, if there is indeed a more significant extent of $\mathrm{Cu}$ oxidation taking place in this system. Whilst deriving new potentials for $\mathrm{Cu}^{+}$and $\mathrm{Cu}^{2+}$ is outside the scope of the present work, an IP approach considering different $\mathrm{Cu}$ oxidation states would additionally be of interest to afford comparison with previous computational QM/ MM studies which found oxidised $\mathrm{Cu}$ to play a key role in $\mathrm{Cu}$ cluster growth by functioning as an anchor site, in particular at surface Zn vacancy sites. ${ }^{26,28}$

\section{Conclusions}

The IP global optimisation approach employed in the present work is largely successful in identifying low-energy structures for $\mathrm{Cu}$ cluster growth upon different $\mathrm{O}$ - and $\mathrm{Zn}$-terminated reconstructed polar $\mathrm{ZnO}$ surfaces, as largely verified by the DFT refinement. Only the O-rich Zn-terminated reconstructed surface posed a significant challenge, which we conclude is a result of electron transfer, namely from adsorbed $\mathrm{Cu}$ to undercoordinated surface $\mathrm{O}$ adatoms which are not fully reduced, which is not well-accounted for by the IP model applied. Hence, future studies will consider applying a similar methodology including potentials for $\mathrm{Cu}^{+}$and $\mathrm{Cu}^{2+}$, in order to be able to conduct a more effective screening of potential $\mathrm{Cu}$ cluster adsorption geometries. Nonetheless, for all four of the reconstructed surfaces investigated, IP and DFT structures largely agreed upon typical $\mathrm{Cu}$ cluster morphologies, with planar $\mathrm{Cu}$ cluster growth being favoured for Zn-terminated reconstructions, whilst 3D Cu cluster growth was observed for the O-terminated reconstructed surfaces. Whilst at first sight these findings do not appear to agree with previous experimental STM studies, upon closer examination it is clear that the discrepancies are probably a consequence of the differences in the size of the clusters modelled in the current study compared with those investigated in the experimental work. Moreover, the rationalisation for the observed behaviour, namely that a strong $\mathrm{Cu}-\mathrm{Zn}$ attractive interaction and $\mathrm{Cu}-\mathrm{O}$ repulsive interactions are responsible for the observed $\mathrm{Cu}$ cluster morphology, is consistent with the rationalisations proposed for previous experimental studies. Hence, the results obtained in the present work corroborate the previous STM experimental studies and illustrate how surface features of the reconstructed polar $\mathrm{ZnO}$ surfaces can affect the morphology of adsorbed $\mathrm{Cu}$ clusters of different sizes. The IP-calculated $\mathrm{Cu}$ cluster adsorption energies show that $\mathrm{Cu}$ clusters adsorbed on the O-poor O-terminated surface, and the O-rich Zn-terminated surface, are especially stable, with highly exothermic adsorption energies, although for $\mathrm{Cu}$ clusters adsorbed on all four $\mathrm{ZnO}$ reconstructed surfaces investigated, the attractive interaction between $\mathrm{Cu}$ and the $\mathrm{ZnO}$ surface means that all of the investigated $\mathrm{Cu}$ cluster morphologies are potentially feasible.

The present work represents a detailed and thorough investigation of the growth and morphology of $\mathrm{Cu}$ clusters over 
reconstructed polar $\mathrm{ZnO}$ surfaces, providing additional evidence for the efficacy of the IP global optimisation approach used in this work and in related previous computational studies, and revealing new insights into the structure of $\mathrm{Cu}$ clusters on $\mathrm{ZnO}$ surfaces that is complementary to existing experimental studies. Furthermore, the $\mathrm{Cu} / \mathrm{ZnO}$ structures obtained in the present work are an ideal starting point for future computational investigations exploring key processes, such as $\mathrm{CO}_{2}$ adsorption and activation, and subsequent catalytic hydrogenation processes, providing model catalysts that exhibit interesting surface features, especially at the $\mathrm{Cu} / \mathrm{Zn}$ interface, that could play an important role in the behaviour of the industrial $\mathrm{Cu} / \mathrm{ZnO}$ catalyst used for methanol synthesis. We intend that this comprehensive and exhaustive computational study will provide valuable insights into the role of support surface structure in determining metal nanoparticle growth, and thus continue the drive towards a more complete and accurate model for complex multi-component catalyst materials.

\section{Conflicts of interest}

There are no conflicts to declare.

\section{Acknowledgements}

This work was supported through the Global Challenges Research Fund (GCRF) through Science \& Technology Facilities Council (STFC), grant number ST/R002754/1: Synchrotron Techniques for African Research and Technology (START). Additionally, the authors acknowledge the support of the Supercomputing Wales project, which is part-funded by the European Regional Development Fund (ERDF) via Welsh Government, STFC Scientific Computing Department's SCARF cluster, and the UK National Supercomputing Service ARCHER (accessed via membership of the Materials Chemistry Consortium which is funded by EPSRC (EP/L000202)), for the provision of computational resources. The UK Catalysis Hub is thanked for resources and support provided via membership of the UK Catalysis Hub Consortium and funded by EPSRC (Grants EP/R026815/1).

\section{References}

1 K. C. Waugh, Catal. Lett., 2012, 142, 1153-1166.

2 G. C. Chinchen, P. J. Denny, D. G. Parker, M. S. Spencer and D. A. Whan, Appl. Catal., 1987, 30, 333-338.

3 G. C. Chinchen, P. J. Denny, J. R. Jennings, M. S. Spencer and K. C. Waugh, Appl. Catal., 1988, 36, 1-65.

4 M. Bowker, R. A. Hadden, H. Houghton, J. N. K. Hyland and K. C. Waugh, J. Catal., 1988, 109, 263-273.

5 D. Waller, D. Stirling, F. S. Stone and M. S. Spencer, Faraday Discuss. Chem. Soc., 1989, 87, 107-120.

6 M. M. Günter, T. Ressler, R. E. Jentoft and B. Bems, J. Catal., 2001, 203, 133-149.

7 B. L. Kniep, T. Ressler, A. Rabis, F. Girgsdies, M. Baenitz, F. Steglich and R. Schlögl, Angew. Chem., Int. Ed., 2004, 43, 112-115.
8 T. Ressler, B. L. Kniep, I. Kasatkin and R. Schlögl, Angew. Chem., Int. Ed., 2005, 44, 4704-4707.

9 M. Behrens, F. Studt, I. Kasatkin, S. Kuhl, M. Havecker, F. Abild-Pedersen, S. Zander, F. Girgsdies, P. Kurr, B.-L. Kniep, M. Tovar, R. W. Fischer, J. K. Norskov and R. Schlogl, Science, 2012, 336, 893-897.

10 M. Behrens, I. Kasatkin, S. Kühl and G. Weinberg, Chem. Mater., 2010, 22, 386-397.

11 J. P. Breen and J. R. H. Ross, Catal. Today, 1999, 51, 521-533.

12 G. C. Shen, S. I. Fujita, S. Matsumoto and N. Takezawa, J. Mol. Catal. A: Chem., 1997, 124, 123-136.

13 F. Studt, M. Behrens and F. Abild-Pedersen, Catal. Lett., 2014, 144, 1973-1977.

14 M. S. Spencer, Top. Catal., 1999, 8, 259-266.

15 D. Mora-Fonz, T. Lazauskas, M. R. Farrow, C. R. A. Catlow, S. M. Woodley and A. A. Sokol, Chem. Mater., 2017, 29, 5306-5320.

16 M. Capdevila-Cortada and N. López, Nat. Mater., 2017, 16, 328-334.

17 M. Bowker, H. Houghton, K. C. Waugh, T. Giddings and M. Green, J. Catal., 1983, 84, 252-255.

18 M. Grunze, W. Hirschwald and D. Hofmann, J. Cryst. Growth, 1981, 52, 241-249.

19 L. V. Koplitz, O. Dulub and U. Diebold, J. Phys. Chem. B, 2003, 107, 10583-10590.

20 O. Dulub, M. Batzill and U. Diebold, Top. Catal., 2005, 36, 65-76.

21 D. Mora-Fonz, T. Lazauskas, S. M. Woodley, S. T. Bromley, C. R. A. Catlow and A. A. Sokol, J. Phys. Chem. C, 2017, 121, 16831-16844.

22 M. L. Paleico and J. Behler, J. Chem. Phys., 2020, 153(5), 054704.

23 S. A. French, A. A. Sokol, S. T. Bromley, C. R. A. Catlow, S. C. Rogers, F. King and P. Sherwood, Angew. Chem., 2001, 113, 4569-4572.

24 C. R. A. Catlow, S. A. French, A. A. Sokol, M. Alfredsson and S. T. Bromley, Faraday Discuss., 2003, 124, 185-203.

25 S. A. French, A. A. Sokol, S. T. Bromley, C. R. A. Catlow and P. Sherwood, Top. Catal., 2003, 24, 161-172.

26 S. T. Bromley, S. A. French, A. A. Sokol, C. R. A. Catlow and P. Sherwood, J. Phys. Chem. B, 2003, 107, 7045-7057.

27 C. R. A. Catlow, S. A. French, A. A. Sokol and J. M. Thomas, Philos. Trans. R. Soc., A, 2005, 363, 913-936.

28 S. A. French, A. A. Sokol, C. R. A. Catlow and P. Sherwood, J. Phys. Chem. C, 2008, 112, 7420-7430.

29 L. Martínez-Suárez, J. Frenzel and D. Marx, Phys. Chem. Chem. Phys., 2014, 16, 26119-26136.

30 B. Yang, C. Liu, A. Halder, E. C. Tyo, A. B. F. Martinson, S. Seifert, P. Zapol, L. A. Curtiss and S. Vajda, J. Phys. Chem. C, 2017, 121, 10406-10412.

31 G. Shi, Q. Chen, Q. Zhang, W. Cai, Z. Li, S. Zhai, H. Yu, F. Tan and Y. Wang, J. Saudi Chem. Soc., 2020, 24, 42-51.

32 S. M. Woodley, J. Phys. Chem. C, 2013, 117, 24003-24014.

33 M. R. Farrow, Y. Chow and S. M. Woodley, Phys. Chem. Chem. Phys., 2014, 16, 21119-21134.

34 T. Lazauskas, A. A. Sokol and S. M. Woodley, Nanoscale, 2017, 9, 3850-3864. 
35 J. D. Gale, J. Chem. Soc., Faraday Trans., 1997, 93, 629-637. 36 J. D. Gale and A. L. Rohl, Mol. Simul., 2003, 29, 291-341.

37 L. Whitmore, A. A. Sokol and C. R. A. Catlow, Surf. Sci., 2002, 498, 135-146.

38 F. Cleri and V. Rosato, Phys. Rev. B: Condens. Matter Mater. Phys., 1993, 48, 22-33.

39 G. Kresse and J. Furthmüller, Phys. Rev. B: Condens. Matter Mater. Phys., 1996, 54(6), 11169-11186.

40 G. Kresse and J. Furthmüller, Comput. Mater. Sci., 1996, 6, 15-50.
41 P. E. Blöchl, Phys. Rev. B: Condens. Matter Mater. Phys., 1994, 50, 17953-17979.

42 G. Kresse, Phys. Rev. B: Condens. Matter Mater. Phys., 1999, 59, 1758-1775.

43 J. P. Perdew, A. Ruzsinszky, G. I. Csonka, O. A. Vydrov, G. E. Scuseria, L. A. Constantin, X. Zhou and K. Burke, Phys. Rev. Lett., 2008, 100, 1-4.

44 R. Laskowski, P. Blaha and K. Schwarz, Phys. Rev. B: Condens. Matter Mater. Phys., 2003, 67, 075102. 